(C) 2000 Springer-Verlag New York Inc.

\title{
Stable and Unstable Solitary-Wave Solutions of the Generalized Regularized Long-Wave Equation
}

\author{
J. L. Bona, ${ }^{1}$ W. R. McKinney, ${ }^{2}$ and J. M. Restrepo ${ }^{3, *}$ \\ ${ }^{1}$ Department of Mathematics and Texas Institute for Computational and Applied Mathematics, \\ University of Texas, Austin, TX 78712, USA \\ 2 Department of Mathematics, North Carolina State University, Raleigh, NC 27695, USA \\ 3 Mathematics Department and Program in Applied Mathematics, University of Arizona, Tuc- \\ son, AZ 85721, USA \\ e-mail: restrepo@math.arizona.edu
}

Received March 28, 2000; accepted August 24, 2000

Communicated by Thanasis Fokas

Summary. Investigated here are interesting aspects of the solitary-wave solutions of the generalized Regularized Long-Wave equation

$$
u_{t}+u_{x}+\alpha\left(u^{p}\right)_{x}-\beta u_{x x t}=0 .
$$

For $p>5$, the equation has both stable and unstable solitary-wave solutions, according to the theory of Souganidis and Strauss. Using a high-order accurate numerical scheme for the approximation of solutions of the equation, the dynamics of suitably perturbed solitary waves are examined. Among other conclusions, we find that unstable solitary waves may evolve into several, stable solitary waves and that positive initial data need not feature solitary waves at all in its long-time asymptotics.

Key words. BBM equation, generalized BBM equation, RLW equation, generalized RLW equation, stable solitary-waves, unstable solitary-waves

\footnotetext{
* JMR was supported by an appointment to the Distinguished Postdoctoral Research Program sponsored by the U.S. Department of Energy, Office of University and Science Programs, and administered by the Oak Ridge Institute for Science and Education. The work of WRM was performed at Argonne National Laboratory as a Faculty Research Participant. JLB was partially supported by the National Science Foundation and the W. M. Keck Foundation. The authors thank B. Lucier for supplying a computer code, parts of which were adapted to produce the solver used in this study. We also thank Michael Weinstein for his helpful comments on this study.
} 


\section{Introduction}

The Regularized Long-Wave equation

$$
u_{t}+u_{x}+u u_{x}-u_{x x t}=0
$$

where $u=u(x, t)$ is a real-valued function of two real variables $x$ and $t$ and subscripts connote partial differentiation, was first put forward as a model for small-amplitude long waves on the surface of water in a channel by Peregrine [1], [2], and later by Benjamin et al. [3]. In physical situations such as unidirectional waves propagating in a water channel, long-crested waves in near-shore zones, and many others, the Regularized Long-Wave equation (RLW equation henceforth) serves as an alternative model to the Kortewegde Vries equation (KdV equation) (see [4], [5]). Among other attractive features, the RLW equation has a linearized dispersion relation more closely matching that of the full Euler equations describing the two-dimensional motion of the free-surface oscillations of an ideal liquid under the force of gravity. It is also considerably easier to numerically integrate (cf. [6], [7], [8]), but does not feature the inverse-scattering theory nor the infinite collection of polynomial conservation laws that obtain for the $\mathrm{KdV}$ equation (see [9]).

Equation (1) and its $\mathrm{KdV}$ relative are typically derived under the assumption that the nonlinear, dispersive media being modeled features small-amplitude, long-wavelength disturbances. The term $u u_{x}=\frac{1}{2} \partial_{x}\left(u^{2}\right)$ models nonlinear effects while $-u_{x x t}$ accounts for the frequency dispersion effects due to long, but finite wavelengths. As explained in Benjamin et al. [3, §2], there are situations where nonlinearity first appears at higher order than quadratic (such as the $\mathrm{mKdV}$ equation $u_{t}+u_{x}+u^{2} u_{x}+u_{x x x}=0$, which arises naturally in modeling the evolution of certain internal waves and in modeling waves in a crystalline lattice [10], [11]). This gives impetus to the study of the generalized-RLW equation (gRLW equation henceforth),

$$
u_{t}+u_{x}+\alpha\left(u^{p}\right)_{x}-\beta^{2} u_{x x t}=0 .
$$

Here $p$ is a positive integer and $\alpha$ and $\beta$ are positive constants. Of course, both $\alpha$ and $\beta$ can be scaled out by a suitable change of variables.

Equation (2) arises in another interesting respect. Attention has been drawn to a different generalization of the RLW equation, namely,

$$
u_{t}+u_{x}+f(u)_{x}+L u_{t}=0,
$$

with $f: \mathbb{R} \rightarrow \mathbb{R}$ typically a polynomial and $L$ a Fourier multiplier operator defined by

$$
\widehat{L v}(\xi)=l(\xi) \hat{v}(\xi)
$$

where the circumflexes connote Fourier transforms and the symbol $l$ of $L$ need not be quadratic as in (1). Equations of the form in (3) or their $\mathrm{KdV}$-analogues arise in a wide variety of circumstances (cf. Benjamin [12], [13], [14]; Saut [15], [16]; Albert et al. [17]; Bona [18], [19]; Abdelouhab et al. [20]). The symbol $l$ reflects the approximation to the linearized dispersion relation that originates from more complete equations of motion. In attempting to understand the interaction between nonlinearity and dispersion, one is naturally led to study (3) for different strengths of the symbol $l$ (e.g. $l(\xi)=|\xi|^{q}$ for 
various values of $q>0$ ). This is a situation where the dispersion may vary in strength while the nonlinearity is fixed. It is natural to suppose that a similar understanding might be gained by studying (2), which features a fixed dispersion relation and varying strength nonlinearity.

One of the interesting features of wave equations that account for nonlinearity and dispersion, but which ignore dissipative effects is that they often possess solitary-wave solutions. Solitary waves are traveling-wave solutions $u(x, t)=\phi(x-c t), c>0$, say, where $\phi$ is typically a smooth function, symmetric about its maximum excursion, or crest, and decaying rapidly to zero away from its crest. For equations like the KdV equation and the mKdV equation whose solutions admit an Inverse-Scattering Transform (IST) representation (cf. [21]), it is known that solitary-wave solutions play a distinguished role in the long-time evolution of a general class of disturbances. For the KdV equation, for example, an initial disturbance breaks up into a finite sequence of independently propagating solitary waves followed by a dispersive tail. In particular, from any disturbance of elevation $(u(x, 0) \geq 0$ everywhere, $u(x, 0) \not \equiv 0)$, at least one solitary wave will emerge in the associated solution of the $\mathrm{KdV}$ equation. Even for model equations like the RLW equation (1) that do not appear to possess an Inverse-Scattering theory, the initial-value problem posed on the whole real line still has the property that initial disturbances resolve into solitary waves and a dispersive tail (see Bona et al. [5], [8], [22]).

We do not at present have a satisfactory theory for why the property of resolution into solitary waves is so ubiquitous. It is suggested here and elsewhere (cf. [23]) that resolution into solitary waves is related to the stability of individual solitary waves under perturbations in the initial data. To be more precise about both these notions, let $\left\{\phi_{c}\right\}_{c>1}$ be a smooth branch of solitary-wave solutions of a nonlinear, dispersive, wave equation like that shown in (3), parameterized by its phase speed $c$. Thus $u(x, t)=\phi_{c}(x-c t)$ is an exact, traveling-wave solution of (3). Such branches are known to exist for a reasonably broad class of nonlinearities $f$ and dispersion operators $L$ (see Weinstein [24]; Benjamin et al. [25]; Albert et al. [26], [17]; Albert [27]; Angulo [28]; and Chen \& Bona [29]). Following Lax in his seminal paper [30], we say that an equation has the property of resolution into solitary waves if, for suitably restricted data $\psi$ (e.g. $\psi$ lies in an $L_{2}$-based Sobolev class like $H^{k}(\mathbb{R})$ for large enough $k$ ), there are phase speeds $c_{i}>1$ and phase translations $\theta_{i}(t), 1 \leq i \leq K$, such that if $u$ is the solution corresponding to $\psi$, then

$$
\left.\begin{array}{l}
u(x, t)=\sum_{i=1}^{K} \phi_{c_{i}}\left(x-c_{i} t+\theta_{i}(t)\right)+r(x, t), \quad \text { where } \\
\lim _{t \rightarrow \infty} \sup _{x \in \mathbb{R}}|r(x, t)|=\lim _{t \rightarrow \infty}|r(\cdot, t)|_{\infty}=0, \quad \text { and } \\
\lim _{t \rightarrow \infty} \theta_{i}(t)=\theta_{i}(\infty)=\mathrm{constant}, \quad 1 \leq i \leq K .
\end{array}\right\}
$$

It may be the case that there are no solitary waves emerging, in which case $K=0$. One can make more detailed requirements about the remainder $r$ based on what we know about the long-time asymptotics of dispersive wave trains, but this aspect will not concern us here. Note that while the $L_{\infty}$-norm of $r$ evanesces as $t$ grows unboundedly, its energy norm (e.g. $L_{2}$-norm for $\mathrm{KdV}, H^{1}$-norm for gRLW) will typically approach a nonzero, constant value.

On the other hand, orbital stability of an individual solitary wave relative to perturbations in the initial data was discussed already by Benjamin [31]. Let $X$ be a Banach space of real-valued functions of a real variable. We measure the size of a wave profile $u(x, t)$ at a fixed time $t$ by the norm $\|\cdot\|_{X}$ on $X$. (In practice, $X$ is usually the energy space for 
the evolution equation which is related to the symbol $l$ of the dispersion operator. Thus, $X=H^{1}(\mathbb{R})$ for the gRLW equation.) The solitary wave $\phi_{c}$ is orbitally stable in the norm on $X$ if given $\epsilon>0$, there corresponds a $\delta>0$ such that if $\psi \in X$ and

$$
\left\|\phi_{c}-\psi\right\|_{X} \leq \delta
$$

then

$$
\inf _{y \in \mathbb{R}}\left\|\phi_{c}(\cdot+y)-u(\cdot, t)\right\|_{X} \leq \epsilon
$$

for all $t \geq 0$, where $u$ is the solution of the relevant initial-value problem corresponding to initial data $\psi$. Since the set $\left\{\phi_{c}(\cdot+y)\right\}_{y \in \mathbb{R}}$ comprises exactly the complete orbit of the solitary wave $\phi_{c}$, this notion corresponds to the usual idea of orbital stability in dynamical systems theory. Of course, it is important that the initial-value problem with initial data in $X$ be well-posed. The stability theory for solitary-wave solutions of KdV-type and RLW-type equations such as (3) has a 25-year history, starting with the very original work of Benjamin [31] (see also Bona [32]). The recent paper [23] has a review of the theory together with a long bibliography.

For the generalized-KdV equation ( $\mathrm{gKdV}$ equation)

$$
u_{t}+u_{x}+\left(u^{p}\right)_{x}+u_{x x x}=0
$$

the theory in [33] (see also [24]) shows that the solitary-wave solutions $\left\{\phi_{c}\right\}_{c>1}$ are stable for any value of the phase speed if $p<5$, and unstable for all values of $c$ if $p \geq 5$. For the stable range, $p=2,3$, and 4 , it is observed that initial data resolves into solitary waves and a dispersive tail as described in (4). This follows from the IST-theory for $p=2,3$ and is observed to be the case in extensive numerical experiments for $p=4$ (see [34] and [35]). For small perturbations of solitary waves, the result has been rigorously established in the work of Pego and Weinstein [36] for $p=4$. For the unstable range $p>5$, numerical simulations indicate that small perturbations of a solitary wave not only leave a neighborhood of the solitary wave's orbit, but in fact blow up in $L_{\infty^{-}}$ norm in finite time (see [34], [37], and the references contained therein). This property that perturbations of solitary waves lead to the formation of singularities has been called strong instability in recent papers of Liu [38], [39]. We adopt this terminology here. It is worth noting that the $\mathrm{gKdV}$ equation with $p \geq 5$ is globally well-posed for data that is small in $H^{1}(\mathbb{R})$, but numerical simulations show that large initial data give rise to solutions that form singularities by first beginning the process of resolution into solitary waves, following which the leading solitary wave goes unstable and blows up.

It is the principal purpose of this paper to bring our collective understanding of the solitary-wave solutions of the gRLW equation to something like the level obtained for the $\mathrm{gKdV}$ equation. The analytical work that guides the numerical study to follow is now explained.

The solitary-wave solutions of the gRLW equation corresponding to a fixed phasespeed $c>1$ have the explicit form

$$
\phi_{c}(x, t)=A\left\{\operatorname{sech}^{2}\left[K\left(x+x_{0}-c t\right)\right]\right\}^{1 /(p-1)},
$$

where

$$
K=\left(\frac{p-1}{2 \beta}\right) \sqrt{\frac{c-1}{c}}, \quad A=\left[\frac{(p+1)(c-1)}{2 \alpha}\right]^{1 /(p-1)}
$$


and $x_{0}$ is any real constant. If $p$ is odd, $-\phi_{c}$ is also a travelling-wave solution. Unlike the situation explained above for the gKdV equation, the initial-value problem for (3) is globally well-posed for initial data of unrestricted size, for all $p \geq 1$. Indeed, if the initial data lies in $H^{1}(\mathbb{R})$, the square of the $H^{1}$-norm,

$$
V(u)=\frac{1}{2} \int_{-\infty}^{\infty}\left[u(x, t)^{2}+\beta^{2} u_{x}(x, t)^{2}\right] d x,
$$

is an invariant of the motion. Thus if $u(x, 0)=\psi(x)$ lies in $H^{1}(\mathbb{R})$, the solution of (3) emanating from $\psi$ has the property that $V(u(\cdot, t))=V(\psi)$ for all $t$ for which the solution exists. This in turn means that

$$
\max _{x \in \mathbb{R}}|u(x, t)| \leq\left[\frac{1}{4 \beta} V(u(\cdot, t))\right]^{1 / 2}
$$

is uniformly bounded. Hence, finite-time blow-up of $H^{1}$-solutions is precluded, and, indeed, the bound on the $H^{1}$-norm of solutions suffices to conclude that the pure initialvalue problem for (3) is globally well-posed in $H^{s}(\mathbb{R})$ for any $s \geq 1$, for all $p$, whatever the size of the initial disturbances.

The stability theory for the solitary-wave solutions of gRLW is a little more complex than for the gKdV. Albert et al. [40] showed that the solitary-wave solutions (6) above are orbitally stable in $H^{1}(\mathbb{R})$ for $p=2,3$, and 4, and Miller and Weinstein [41] demonstrated asymptotic stability in the same range. Souganidis and Strauss in [42] (hereon referred to as $\mathrm{S} \& \mathrm{~S}$ ) examined the case $p \geq 5$ and drew the following conclusions, which are central to the present study. A second invariant functional,

$$
W(u)=\int_{-\infty}^{\infty}\left[\frac{1}{2} \beta^{2} u_{x}^{2}-\frac{\alpha}{p+1} u^{p+1}\right] d x,
$$

plays an important role in the stability theory for (2). By combining (7) and (8) appropriately, it is seen that

$$
E(u)=-\int_{-\infty}^{\infty}\left[\frac{1}{2} u^{2}+\frac{\alpha u^{p+1}}{p+1}\right] d x
$$

is likewise an invariant functional. Moreover, the linear combination $E+c V$ has the property that its Fréchet derivative $E^{\prime}+c V^{\prime}$ vanishes indentically when evaluated at the solitary wave $\phi_{c}$. The functional

$$
d(c)=E\left(\phi_{c}\right)+c V\left(\phi_{c}\right)
$$

defines what we will call the total energy of the solitary wave $\phi_{c}$. The following two results from $S \& S$ form the basis for the present study. Theorem 2.5 of $S \& S$, specialized to the gRLW equation, states that the solitary wave $\phi_{c}$ is $H^{1}$-unstable if $d^{\prime \prime}(c)<0$ and $H^{1}$-stable if $d^{\prime \prime}(c)>0$. For the gRLW equation, an explicit calculation reveals that

$$
d(c)=b c^{1 / 2}(c-1)^{\sigma+1 / 2},
$$




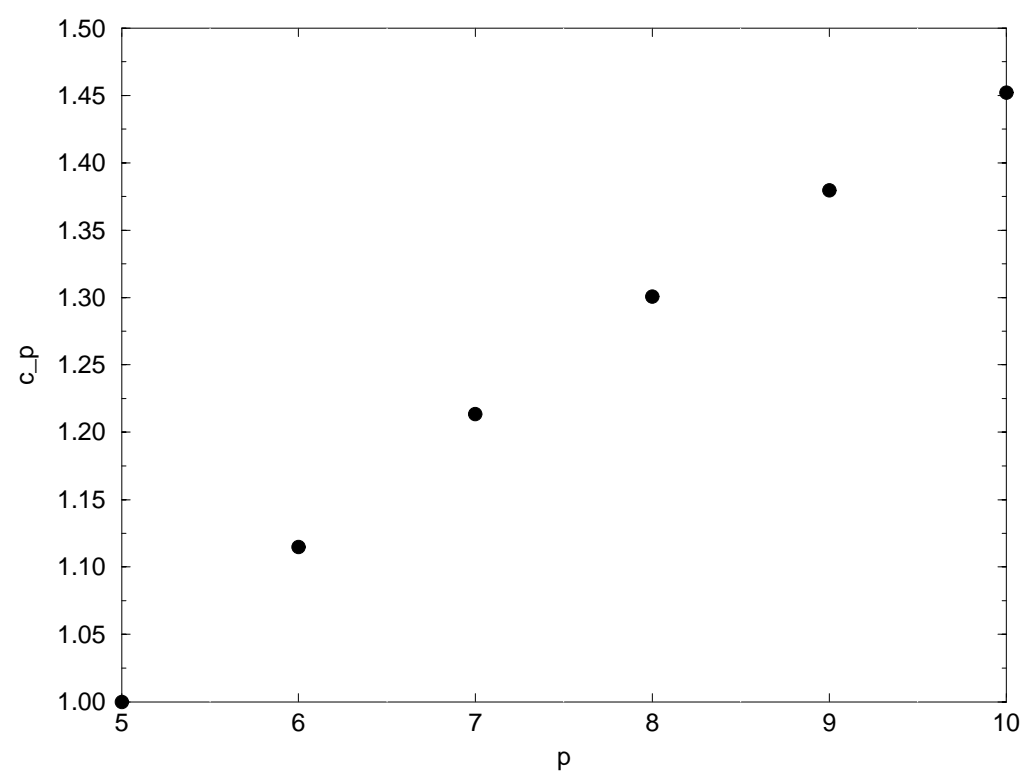

Fig. 1. Critical speed $c_{p}$ as a function of $p$.

where $\sigma=2 /(p-1)$ and $b$ is a positive constant related to the energy of the ground state (cf. [42, p. 208]). A consequence of this is Theorem 3.2 of S\&S, which states (a) if $p \leq 5$, then $\phi_{c}$ is $H^{1}$-stable, and (b) if $p>5$, then $\phi$ is $H^{1}$-stable for $c>c_{p}$, and $H^{1}$-unstable for $1<c \leq c_{p}$, where

$$
c_{p}=\frac{1+\sqrt{2-\sigma^{-1}+2 \sigma^{-1}}}{2(\sigma+1)} .
$$

Note that $c_{p}=1$ when $p=5$ and $c_{p}>1$ for $p>5$. Figure 1 shows how the critical speed $c_{p}$ depends on $p$, while Figure 2 shows a plot of $d^{\prime}(c)$ for $p=8$. In this figure, the unstable regime corresponds to $1<c \leq c_{p}$.

The plan of this paper is as follows. Since the conclusions to be drawn in this study are based on numerical simulations of the evolution equations, a detailed description is presented in Section 2 of the algorithm used for the approximation of solutions. A computer code that implements the algorithm is tested for stability, convergence, and accuracy. Stable solitary waves are examined in Section 3. As the theory predicts, these waveforms are orbitally stable, but we find some interesting and somewhat unexpected conclusions when larger perturbations are introduced. Unstable solitary waves are featured in Section 4. As mentioned before, these waves cannot go over to singularity formation as apparently happens for the $\mathrm{gKdV}$ equations with $p>5$. Instead, we find they deform to stable solitary waves followed by a dispersive wave train. With some effort, we are able to see the fission of an unstable solitary wave into more than one solitary wave. The conclusions reached in Sections 3 and 4 are briefly summarized in Section 5, where we put forward a tentative picture of the long-time asymptotics of solutions of the gRLW equation. 


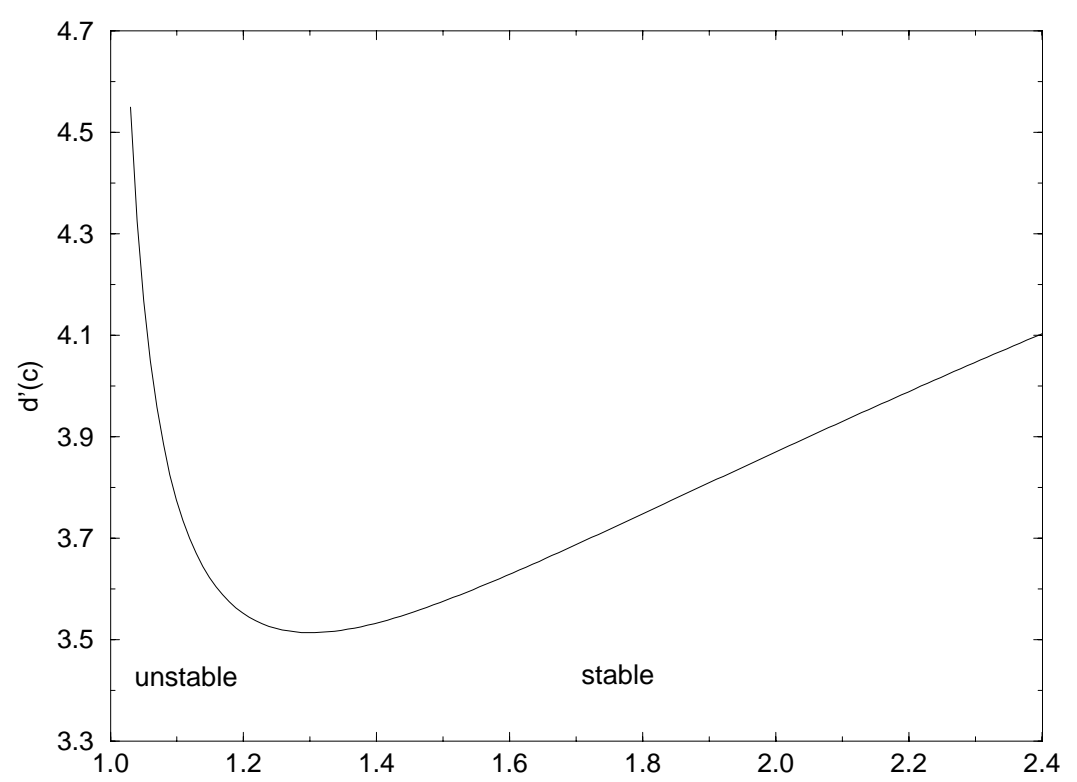

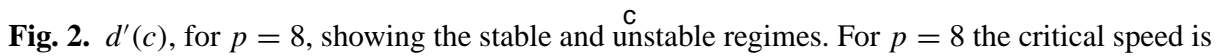
$c_{p}=1.30091$.

\section{Description of the Numerical Technique}

Fourier-spectral methods are standard techniques for the solution of the Regularized Long Wave equation on a spatially periodic domain (cf. [7], [43], [44]). However, our interest lies in solutions to the pure initial-value problem. Hence, if Fourier-spectral techniques are to be employed in the spatial approximation of solutions to the pure initial-value problem

$$
\begin{aligned}
\left(I-\beta^{2} \partial_{x}^{2}\right) u_{t} & =-\partial_{x}(u+\alpha v), \quad \text { for } t \geq 0, \\
u(x, t) & \rightarrow 0 \quad \text { as }|x| \rightarrow \infty, \\
u(x, 0) & =u_{0}(x) \quad \text { for } x \in \mathbb{R},
\end{aligned}
$$

where $v=u^{p}$, there are two aspects with which we must contend. These are the manner in which the nonlinearity is discretized in space, and the matter of discerning under what circumstances, if any, are solutions to the periodic-in-space problem approximate solutions to the pure initial-value problem (11).

One expects that if the solution in question has bounded support or decays to zero at infinity rapidly, then approximating with a periodic problem of sufficiently large period will result in an accurate rendition of the solution, at least on the period domain and over certain time intervals. Theoretical justification for this point of view for a related, fully continuous problem appears in Bona [45] and Guo \& Manoranjan [7], while Pasciak [44] deals with the direct numerical approximation of (11) via Fourier series when $v=u^{2}$.

Pasciak's discussion of the spatial discretization, which involves restricting a function $f$ defined on $\mathbb{R}$ to $\Omega_{\ell}=[0, \ell]$ and then projecting the result onto the finite-dimensional 
space of truncated Fourier series, can be taken over intact for the more general power $v=u^{p}$ appearing here. For a function $g \in L_{2}\left(\Omega_{\ell}\right)$, the usual space of measurable, square-integrable functions defined on $[0, \ell]$, the finite Fourier transform $\hat{g}=\mathcal{F}_{\ell} g$ is an element of $l_{2}(\mathbb{Z})$, the space of square-summable complex vectors, given by

$$
\hat{g}(k)=\mathcal{F}_{\ell} g(k)=\frac{1}{\ell} \int_{0}^{\ell} g(x) e^{-i \frac{2 \pi k x}{\ell}} d x,
$$

$k=\ldots,-1,0,1, \ldots$ The inverse transform is simply the usual Fourier series. Thus, if $h \in l_{2}(\mathbb{Z})$, then

$$
\left(\mathcal{F}_{\ell}^{-1} h\right)(x)=\sum_{k \in Z} h(k) e^{i \frac{2 \pi k x}{\ell}}
$$

for $x \in \Omega_{\ell}$. The mapping $\mathcal{F}_{\ell}$ is a Hilbert-space isomorphism from the closed subspace of $\ell$-periodic elements of $L_{2}\left(\Omega_{\ell}\right)$ onto $l_{2}(\mathbb{Z})$. Suppose (11) is posed as a periodic initialvalue problem instead of as a problem on the whole of $\mathbb{R}$, viz.,

$$
\begin{aligned}
\left(I-\beta^{2} \partial_{x}^{2}\right) \partial_{t} u_{\ell} & =-\partial_{x}\left(u_{\ell}+\alpha v_{\ell}\right), \\
v_{\ell} & =u_{\ell}^{p}, \\
u_{\ell}(x, t) & \rightarrow u_{\ell, 0}(x) \quad \text { for } x \in \Omega_{\ell}, \\
u_{\ell}(0, t) & =u_{\ell}(\ell, t) \quad \text { for } t \in[0, T],
\end{aligned}
$$

where $T>0$ defines the time-interval of interest. This system may be expressed as an infinite system of ordinary differential equations via the Fourier transform in the usual way:

$$
\frac{d}{d t} \hat{u}_{\ell}(k)=P_{\ell}^{-1}(k) Q_{\ell}(k)\left(\hat{u}_{\ell}(k)+\alpha \hat{v}_{\ell}(k)\right)
$$

for all $k \in \mathbb{Z}$, where $P_{\ell}$ and $Q_{\ell}$ are the symbols of the operators $P_{\ell}\left(\partial_{x}\right)=I-\beta^{2} \partial_{x}^{2}$ and $Q_{\ell}\left(\partial_{x}\right)=\partial_{x}$, so, for $k \in \mathbb{Z}$,

$$
P_{\ell}(k)=1+\beta^{2} \frac{4 \pi^{2}}{\ell^{2}} k^{2} \quad \text { and } \quad Q_{\ell}(k)=\frac{2 \pi i}{\ell} k .
$$

As mentioned already, it is expected that solutions $u$ and $u_{\ell}$ of (11) and (12), respectively, will be close on $\Omega_{\ell} \times[0, T]$ for a certain time interval $T$, related inversely to $\ell$, provided that $u$ is essentially zero on $\left(\mathbb{R} \backslash \Omega_{\ell}\right) \times[0, T]$. Pasciak shows [44, Theorem 4.1] that norms of the difference between $u$ and $u_{\ell}$ on $\Omega_{\ell}$ may be bounded in terms of the rate at which $u$ decays and the size of $\ell$. Indeed, if $|u|$ has values below machine accuracy outside $\Omega_{\ell}$ for $0 \leq t \leq T$, then $u-u_{\ell}$ is insignificant on $\Omega_{\ell} \times[0, T]$. It is possible to get a fairly good idea of the time $T$ over which $u-u_{\ell}$ is small on $\Omega_{\ell}$ as a function of $\ell$ and aspects of the initial data by experimental means. The experiments to be reported here are concerned with initial values that feature exponentially decaying tails. In consequence, it is not required to take very large values of $\ell$ to achieve successful approximations, at least over moderate times $T$. In fact, we have found it convenient to fix $\ell=1$ and rescale the spatial variable. This ploy simplifies coding slightly, but does not obviate any real difficulties. The rescaling induces larger gradients in the solutions and this requires 
finer spatial and temporal structure to accurately resolve the solution at the fully discrete level.

The second stage consists of obtaining a semidiscrete approximation to the finitedomain problem by truncation of the Fourier series expansion of the solution. Let $S$ be the linear span of trigonometric polynomials of degree at most $N / 2$, defined as

$$
S=\operatorname{span}\left\{e^{\frac{i 2 \pi k x}{\ell}} \mid-\frac{N}{2} \leq k \leq \frac{N}{2}-1\right\},
$$

and let $\Pi$ be the $L^{2}$-projection onto $S$. An element in $S$ is determined by its values at the points $\Omega_{\ell, N}=\left\{x_{j}=j 2 \ell / N, j=0,1, \ldots, N-1\right\}$. In consequence, the projection $\Pi g$ of a continuous periodic function $g$ may be computed via the discrete Fourier transform

$$
\left(\mathcal{F}_{\ell, N} g\right)_{k}=\frac{1}{N} \sum_{j=0}^{N-1} g\left(x_{j}\right) e^{-i 2 \pi k x_{j} / \ell}, \quad \text { where } k=-\frac{N}{2}, \ldots, \frac{N}{2}-1
$$

whose inverse when restricted to $S$ is

$$
\mathcal{F}_{\ell, N}^{-1} h\left(x_{j}\right)=\sum_{k=-N / 2}^{N / 2-1} h_{k} e^{i 2 \pi k x_{j} / \ell}, \quad \text { with } j=0, \ldots, N-1,
$$

together with the identification between $S$ and the $N$-dimensional complex vector space $S_{N}=\left\{\left(g\left(x_{j}\right)\right)_{j=0}^{N-1}: g \in S\right\}$. Let $\Pi_{N}$ denote the projection followed by the identification of $S$ with $S_{N}$. Projecting the system (11)-(12) onto $S$ and following with the identification between $S$ and $S_{N}$ in the standard way leads to a system of nonlinear ordinary differential equations having the form

$$
\begin{aligned}
\frac{d U}{d t}= & -P_{\ell, N}^{-1}(k) Q_{\ell, N}(k)(U+\alpha V) \\
& \text { for }\left(x_{j}, t\right) \in \Omega_{\ell, N} \times[0, T], \\
U\left(x_{j}, 0\right)= & U_{0}\left(x_{j}\right), \quad j=0,1, \ldots, N-1,
\end{aligned}
$$

with $U \in C^{1}\left([0, T], S_{N}\right)$. The error between the element of $S$ associated with $U(\cdot, t)$ and $u_{\ell}(\cdot, t)$ was shown in [44] to depend on the smoothness of the solution $u_{\ell}$ and on the size of $N$. If the initial data $u_{\ell, 0} \in H^{m}\left(\Omega_{\ell}\right)$ and the element of $S$ corresponding to $U_{0}$ approximates $u_{\ell, 0}$ appropriately, then the error is of order $N^{-m}$, uniformly for $0 \leq t \leq T$.

Since the nonlinear term is a product of the function $u$ with itself $p$ times, it is represented via a convolution of its Fourier counterpart with itself $p$ times; hence there is a very real possibility of significant aliasing errors. To minimize this potential source of error, an effective but computationally expensive strategy was adopted in obtaining the results reported here. The nonlinear term $v$ was handled pseudo-spectrally (cf. [46, pp. 83-85]): As the solution in $S_{N}$ evolves in time, the nonlinear term is computed at each time step by transforming back to $S$, forming the power $u^{p}$, and projecting the result back into $S_{N}$. To avoid aliasing errors, we solved (14) using $N_{p}=p N$ interpolating knots, where $N$ is a large enough number of interpolating knots that $U_{0}$ was accurately represented in $S_{N}$ (this technique is known as zero-padding). Determining whether the initial data is well padded is straightforward since, in all the experiments reported here, 
it was a function with rapid decay to zero at $\pm \infty$. However, this data may evolve into a solution with support that occupies the whole finite interval, and what is adequate zeropadding at the initial time may not be adequate later in the evolution of the solution. To obtain the desired effect from the zero-padding, we over-resolved the solution by making $N$ large, and monitored quantitatively the spectrum of the solution as it evolved in time. The combination of over-resolving and zero-padding by $(p-1) N$ yielded satisfactory results as we will show presently.

The system of ordinary differential equations represented in Eq. (14) is given explicitly by

$$
\begin{aligned}
\frac{d U_{k}}{d t} & =-\left(1+\frac{4 \pi^{2}}{\ell^{2}} \beta^{2} k^{2}\right)^{-1} \frac{2 \pi}{\ell} i k\left(U_{k}+\alpha V_{k}\right), \\
U_{k}(0) & =U_{0, k}, k=-N_{p} / 2 \ldots N_{p} / 2-1,
\end{aligned}
$$

where the vector $(U)_{k=-N / 2}^{N / 2-1}=\mathcal{F}_{\ell, N} u$. In the numerical scheme, the fact that $u$ is realvalued was exploited to permit the use of real discrete Fourier transforms. These transforms were performed using the well-known software package FFTPACK [47].

Since (15) is not stiff, it was integrated forward in time using a variable-order, variable-time step, Adams-Bashforth-Moulton method. The specific ODE solver used was Shampine and Watts's DEABM package (1980 version), which is documented in [48]. The time steps between calls to DEABM were fixed in size to enable better control of the dissipation inherent in the time-stepping scheme. The DEABM code requires that the user specify an absolute error tolerance $\mathrm{ATOL}_{k}$ and a relative error tolerance $\mathrm{RTOL}_{k}$ for each equation, $-N / 2 \leq k \leq N / 2-1$. These tolerances are used by the package in a local error test at each integration step. For each vector component $y_{k}$ of the ODE, the local error test is

$$
\left|\mathrm{e}_{k}\right| \leq \underset{k}{\mathrm{RTOL}}\left|y_{k}\right|+\underset{k}{\mathrm{ATOL}} .
$$

This error test is used by the package to control the size of the time step. We have set $\mathrm{RTOL}_{k}=0$ for all $k$ and use weighted values of $\mathrm{ATOL}_{k}$ in our code, so that the local error test becomes

$$
\left|\mathrm{e}_{k}\right| \leq \frac{\text { tol }}{\left(1+\frac{4 \pi^{2}}{\ell^{2}} \beta^{2} k^{2} / 4\right)^{1 / 2}},
$$

which directly translates into an error bound in the $H^{1}$-norm. The value of tol was fixed to $10^{-10}$ for all the experiments reported in this study.

The reader is reminded that wrap-around of the solution is inherent to the periodicity in the numerical scheme described above. Therefore, in the figures to be shown in later sections of this study, a solitary wave may appear to precede its radiative tail. The wraparound has the potential of causing problems by allowing the solution to interact with its "wake." In the numerical solutions to be presented in this study, we have taken care to avoid cases where this situation would have a significant bearing on the final outcome of the experiments.

To test the accuracy of the numerical scheme, the solution $U=\mathcal{F}_{\ell, N}^{-1}\left(U_{k}\right)$ of (14)(15) was initialized with the projection onto $S$ of the solitary-wave solution in (6), and the result compared with the exact traveling wave. It is helpful to observe the error 
made in numerical simulations of solitary waves in three parts, as follows. Let $\phi=\phi_{c}$ connote the exact solution displayed in (6) as before. It is temporarily convenient to write its amplitude $A$ as $A_{c}$ and to think of $\phi$ as parameterized by $A$. Thus $\phi_{c}=\phi_{A}$ when $A=A_{c}$. Let $\Phi\left(t_{k}\right) \in S$ be the solution of (14)-(15) obtained via the numerical scheme outlined above. The error in the approximation is $\left\|\phi_{c}\left(\cdot-c t_{k}\right)-\Phi\left(t_{k}\right)\right\|$, where various norms on functions defined on $\Omega_{\ell}$ might be of interest. Let $A_{k}=\left\|\Phi\left(t_{k}\right)\right\|_{\infty}$ be the amplitude of the computed approximation. The relative amplitude error at time $t_{k}$ in the simulation is defined to be

$$
\frac{\left|A_{c}-A_{k}\right|}{A_{c}}
$$

Let $\bar{\theta}$ be such that

$$
\min _{0 \leq \theta \leq \ell}\left\|\phi_{A_{k}}\left(\cdot-c t_{k}-\theta\right)-\Phi\left(t_{k}\right)\right\|
$$

is taken on at $\bar{\theta}$. The quantity in (17) is called the shape error. It is a measure of by how much $\Phi$ deviates from a true solitary wave when adjustment is made for the amplitude and the phase errors. Finally, the phase error at the $k^{\text {th }}$ time step is simply

$$
\sigma_{k}=\bar{\theta}-c t_{k} \text {. }
$$

By the triangle inequality,

$$
\begin{aligned}
\text { total error }= & \left\|\phi_{c}\left(\cdot-c t_{k}\right)-\Phi\left(t_{k}\right)\right\| \\
\leq & \left\|\Phi\left(t_{k}\right)-\phi_{A_{k}}\left(\cdot-c t_{k}-\bar{\theta}\right)\right\| \\
& +\left\|\phi_{A_{k}}\left(\cdot-c t_{k}-\bar{\theta}\right)-\phi_{A_{c}}\left(\cdot-c t_{k}-\bar{\theta}\right)\right\| \\
& +\left\|\phi_{c}(\cdot+\sigma)-\phi_{c}(\cdot)\right\| .
\end{aligned}
$$

The first term on the right-hand side of (19) is the shape error, the second term is proportional to the amplitude error, and the third term is proportional to the phase error (at least for small values of the latter two quantities). This scheme for the analysis of the numerical errors made in propagating a traveling wave is taken from [4], [8].

Figure 3 and Table 2 give these errors for a number of representative cases. This data conveys the high performance characteristics of the numerical scheme. The physical parameters had the following values: $\alpha=\beta=1.0, p=8$, and the final time $T=2$; the solitary-wave speed was $c=2$. The diagnostic integration step and order, reported by DEABM as a function of $N$, is given in Table 1 . The invariant functionals given by (8) and (9) were also monitored for accuracy. In the fully discrete approximation with $N=4096$, the resolution used in the experiments appearing in the rest of this study, these quantities were found to be constant to seven decimal places.

Some of the experiments to be shown in later sections demanded high resolution, which in turn put severe restrictions on the size of the time step. In a number of these experiments, the object of our attention was not the solitary wave itself, but rather, some other feature of the solution. Some of these features were difficult to assess because of their relative size as compared to the solitary wave, or because of interference between competing features. To resolve these features of the solution without interference from the main solitary-wave, a "subtraction" algorithm was devised. When invoked at some 
Table 1. Order, time step, and discretization points $N$ as reported by DEABM diagnostics at $t=2$ for the integration of solitary-wave data, with $p=8, c=2.0$.

\begin{tabular}{ccr}
\hline Order & Step Size & \multicolumn{1}{c}{$N$} \\
\hline 11 & 0.0101 & 512 \\
12 & 0.0078 & 1024 \\
11 & 0.0112 & 2048 \\
11 & 0.0081 & 4096 \\
\hline
\end{tabular}

particular time $t$, the algorithm starts by identifying the main solitary-wave and its maximum value on the discrete grid. Twenty points on either side of the location of the discrete maximum were then used to carry out a cubic spline interpolation of this solitary wave, resulting in a highly accurate estimation of its position and amplitude. Finally, an analytical solitary wave of the estimated amplitude and position is subtracted from the solution. The numerical integration of the remainder was then continued.

The subtraction algorithm was deemed satisfactory if the difference between the solution and the solution without the solitary wave, at some specific later time, was small when allowance was made for the absence of the solitary wave. To assess the algorithm's effectiveness, we performed an experiment in which runs with the same initial data (corresponding to an unstable case, see Section 4) were compared at $t=4.00$. All the runs had identical parameter values. In the first run (run $R_{1}$ ), the leading solitary wave was subtracted at $t=1.00$. In the second run $\left(R_{2}\right)$ and the third run $\left(R_{3}\right)$, the leading solitary waves were subtracted at $t=0.96$ and $t=1.12$, respectively. Taking run $R_{1}$ as a reference, the absolute difference over the whole domain was computed. Representing the maximum norm of the differences symbolically as $\left|R_{1}-R_{2}\right|$ and $\left|R_{1}-R_{3}\right|$, it was found that $\left|R_{1}-R_{2}\right|=1.1394 \times 10^{-3}$ and $\left|R_{1}-R_{3}\right|=1.0414 \times 10^{-3}$, respectively. This experiment illustrates the efficacy of the subtraction algorithm at a level suitable for our purposes.

In the following two sections, this numerical scheme will be used to approximate solitary-wave solutions of the gRLW equation. Interest will center on the evolution of

Table 2. Errors, as a function of $N$ at $T=2$. Solitary-wave initial data, with $p=8$, $c=2.0$. In the calculations, with $p=8$, the actual resolution is $N_{p}=8 N$, and hence the grid size $h=2 \pi / N_{p}$. The time step and order of the integrator, as a function of $N$, appear in Table 1 .

\begin{tabular}{rcrr}
\hline \multicolumn{1}{c}{$N$} & Amplitude Error & \multicolumn{1}{c}{ Phase Error } & \multicolumn{1}{c}{ Shape Error } \\
\hline 4096 & $0.56157633113 \mathrm{E}-10$ & $0.24600197435 \mathrm{E}-08$ & $0.51929011036 \mathrm{E}-10$ \\
2048 & $0.25260995988 \mathrm{E}-08$ & $0.11533584221 \mathrm{E}-06$ & $0.24145409028 \mathrm{E}-08$ \\
1024 & $0.38543251083 \mathrm{E}-05$ & $-0.16559321581 \mathrm{E}-05$ & $0.27148755561 \mathrm{E}-05$ \\
512 & $0.12613054358 \mathrm{E}-02$ & $-0.17950192756 \mathrm{E}-01$ & $0.92594491223 \mathrm{E}-03$ \\
256 & $0.29658736279 \mathrm{E}-01$ & $-0.12670361685 \mathrm{E}+01$ & $0.38103659781 \mathrm{E}-01$ \\
\hline
\end{tabular}



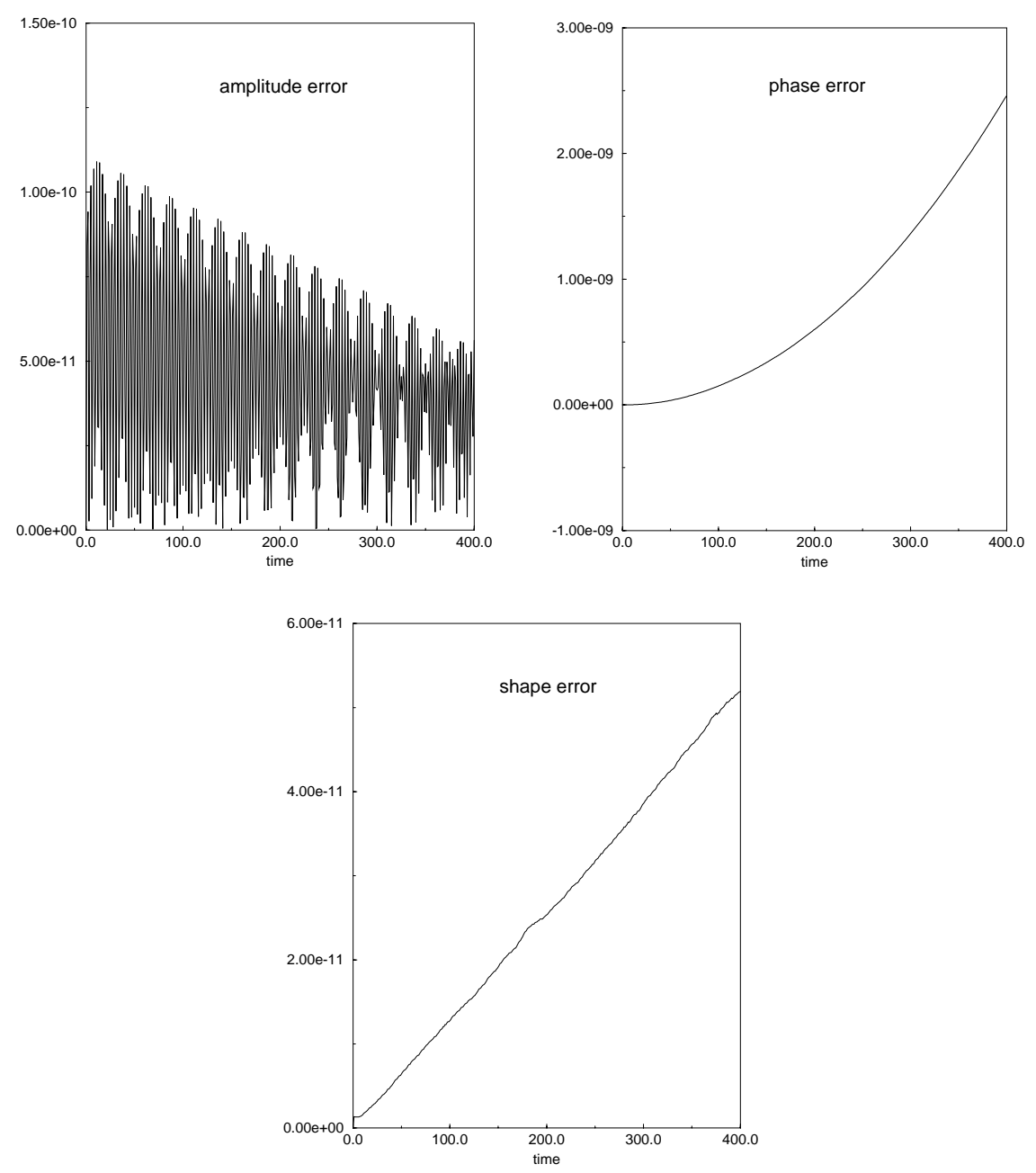

Fig. 3. Amplitude, phase, and shape error. Comparison to exact solution, with $c=2, \alpha=\beta=1$, and $p=8, N=4096$.

solitary-wave solutions for $p>5$ in both the stable and unstable regime of parameters. In Section 3, attention is concentrated on solutions corresponding to perturbations of orbitally stable solitary waves, while Section 4 features experiments with unstable solitary waves. The perturbations considered are simple amplitude and width scalings.

In what follows, the term "exact solution" refers to analytically exact solutions of the differential equation, whereas "solution" refers to the output of the numerical scheme. The distinction between the analytically exact speed $c$ and the observed, asymptotic speed of the numerical solution is made clear by denoting the latter $c^{*}$. A similar convention is taken to distinguish the amplitudes $A$ and $A^{*}$. Unless otherwise noted, the solutions correspond to $\alpha=\beta=1$, and were computed using $N=4096$. The actual number of discretization points was always $N_{p}=p N$. 


\section{Experiments with Stable Solitary Waves}

In this section, the evolution of stable solitary waves subject to amplitude and width perturbations is considered. In the actual computation, a spatial scaling parameter is introduced, namely $x \rightarrow 0.005 x$. The scaling parameter affects the amplitude, the speed, and the energy of the solution. On the other hand, all graphs and numerical data from the experiments are reported in unscaled variables. The introduction of the scaling parameter has little relevance to the experiments and results presented in this section, but will play a role in the preparation of initial data in experiments to be reported in Section 4. As a reminder, for $p=8$, the critical speed is $c_{p}=1.300914176$ corresponding to a critical amplitude $A=1.044258166$. The $H^{1}$-norm, which is also the square root of the invariant functional $V$ introduced in (7), will be used as a diagnostic tool in the experiments. The $H^{1}$-norm of a solitary wave of the form given in (6) has an exact analytic expression which allows its value to be determined numerically to very high accuracy (cf. [49, p. 344]).

In the first instance, the initial data is a perturbed solitary wave, viz.

$$
U(x, 0)=\gamma \phi_{c}(x)
$$

where $\gamma$ is a real parameter, $c>c_{p}$, and $\phi_{c}$ is as presented in (6). The positive number $\gamma$ will be referred to as the amplitude perturbation parameter. The solution to (11) with initial data as in (20) will be a traveling-wave solution only when $\gamma=1$. Indeed, elementary phase-plane analysis shows the solutions displayed in (6) and their negatives in case $p$ is odd, to be the only bounded, traveling-wave solutions that tend to zero at $\pm \infty$. When $c>c_{p}$, so that the solitary wave is orbitally stable, then if $\gamma$ is near to 1 -corresponding to a small perturbation - the solution emanating from $\gamma \phi_{c}(x)$ proves to resolve into a single, stable solitary wave. For considerably greater values of $\gamma$, we still observed the emergence of a stable solitary wave, followed by a substantial dispersive tail. However, for fixed amplitude or speed and for $\gamma$ considerably less than 1 , the solution was observed to go over to a purely dispersive wave form. This phenomenon, which will be depicted presently, seemed sufficiently interesting that a series of experiments was performed to determine what appears to be a basin for attraction for the one-parameter family of solitary waves. More precisely, for a selected set of values of $c>c_{p}$, the corresponding value $\tilde{\gamma}=\tilde{\gamma}(c)$ was determined, to three decimal places, as the demarcation between resolution into a solitary-wave profile and resolution into high-frequency dispersion. Thus, for values $\gamma<\tilde{\gamma}$, the numerical integration of the equation yielded a purely dispersive solution.

These experiments are now described in some detail. Table 3 shows a typical set of experiments leading to approximate values $\gamma^{*}$ of $\tilde{\gamma}=\tilde{\gamma}(c)$. For $p$ fixed, long-time runs are reported with initial data $u_{0}(x)=\gamma \phi_{c}(x)$, where $c>c_{p}$ is fixed at selected values and $\gamma$ is varied. For $\gamma>\gamma^{*}$, we record the observed speed $c^{*}$ and amplitude $A^{*}$ of the emergent solitary wave. Even a cursory study of this data makes a convincing case for the existence of the function $\tilde{\gamma}(c)$.

Because of the definition of $d(c)$ and the fact noted earlier that

$$
E^{\prime}\left(\phi_{c}\right)+c V^{\prime}\left(\phi_{c}\right)=0
$$


Table 3. Evolution of perturbed stable solitary-wave solutions with speed $c$ and $\gamma<1$. The speed $c^{*}$ and amplitude $A^{*}$ of the emergent solitary wave at time $T=12.50$ is recorded when such a wave appears. The actual initial amplitude of the wave was $A$.

\begin{tabular}{|c|c|c|c|c|c|}
\hline$c$ & $A$ & $\gamma$ & Outcome & $c^{*}$ & $A^{*}$ \\
\hline 1.500 & 1.123 & $\begin{array}{l}0.998 \\
0.996 \\
0.994 \\
0.993 \\
0.992 \\
0.990 \\
0.950\end{array}$ & $\begin{array}{c}\text { stable } \\
\text { stable } \\
\text { stable } \\
\text { stable } \\
\text { disperses } \\
\text { disperses } \\
\text { disperses }\end{array}$ & $\begin{array}{l}1.447 \\
1.413 \\
1.367 \\
1.330\end{array}$ & $\begin{array}{l}1.105 \\
1.092 \\
1.074 \\
1.058\end{array}$ \\
\hline 2.000 & 1.240 & $\begin{array}{l}0.990 \\
0.970 \\
0.962 \\
0.961 \\
0.960 \\
0.950\end{array}$ & $\begin{array}{l}\text { stable } \\
\text { stable } \\
\text { stable } \\
\text { disperses } \\
\text { disperses } \\
\text { disperses }\end{array}$ & $\begin{array}{l}1.804 \\
1.540 \\
1.351\end{array}$ & $\begin{array}{l}1.202 \\
1.135 \\
1.068\end{array}$ \\
\hline 2.500 & 1.314 & $\begin{array}{l}0.940 \\
0.935 \\
0.934 \\
0.933 \\
0.930\end{array}$ & $\begin{array}{c}\text { stable } \\
\text { stable } \\
\text { disperses } \\
\text { disperses } \\
\text { disperses }\end{array}$ & $\begin{array}{l}1.515 \\
1.377\end{array}$ & $\begin{array}{l}1.128 \\
1.078\end{array}$ \\
\hline 3.000 & 1.369 & $\begin{array}{l}0.920 \\
0.912 \\
0.911 \\
0.910 \\
0.900\end{array}$ & $\begin{array}{c}\text { stable } \\
\text { stable } \\
\text { disperses } \\
\text { disperses } \\
\text { disperses }\end{array}$ & $\begin{array}{l}1.582 \\
1.361\end{array}$ & $\begin{array}{l}1.147 \\
1.072\end{array}$ \\
\hline
\end{tabular}

it follows immediately that

$$
d^{\prime}(c)=V\left(\phi_{c}\right)
$$

For purposes of comparison across different values of $c$, it is convenient to define

$$
\begin{aligned}
M_{\tilde{\gamma}}(c) & =V\left(\tilde{\gamma}(c) \phi_{c}\right)=\tilde{\gamma}(c)^{2} V\left(\phi_{c}\right) \\
& =\tilde{\gamma}(c)^{2} d^{\prime}(c) .
\end{aligned}
$$

Table 4 shows the way that $\gamma^{*}$ depends on $c$ via a set of simulations like those featured in Table 3. This table, which has data for $p=8$, shows clearly that $\tilde{\gamma}$ is a decreasing function of $c$. This aspect is typical, and seems to be true for all the values of $p$ we checked. Figure 4 shows graphically the relation between $d^{\prime}(c)=V\left(\phi_{c}\right)$ and an approximation of $M_{\gamma}(c)$ when $p=8$ and also when $p=6$. The first graph is derived from the data in Table 4.

A more careful study of Table 3 reveals that, for $\gamma>\tilde{\gamma}$, the speed and amplitude of the solitary wave emerging from $\gamma \phi_{c}$ is an increasing function of $\gamma$. This result is expected since $V\left(\gamma \phi_{c}\right)$ decreases as $\gamma$ decreases, and hence the upper bound on a possibly 
Table 4. $H^{1}$-norm of solitary waves of initial speed $1.5 \leq c \leq 3.0$, for $p=8$. The quantity $\gamma^{*}(c)$ is the smallest value of $\gamma$ tested for which the final outcome is a solitary wave rather than a purely dispersive waveform.

\begin{tabular}{cccc}
\hline$c$ & $A$ & $H^{1}$-norm & $\gamma^{*}(c)$ \\
\hline 1.5 & 1.122824262 & 3.55013461 & 0.993 \\
1.6 & 1.152453457 & 3.58143811 & 0.987 \\
1.7 & 1.178113742 & 3.61705077 & 0.981 \\
1.8 & 1.200803060 & 3.65029220 & 0.974 \\
1.9 & 1.221178892 & 3.68703631 & 0.968 \\
2.0 & 1.239698493 & 3.72274123 & 0.962 \\
2.1 & 1.256693342 & 3.75693670 & 0.956 \\
2.2 & 1.272411777 & 3.78937192 & 0.950 \\
2.3 & 1.287044892 & 3.82397177 & 0.945 \\
2.4 & 1.300743029 & 3.85675837 & 0.940 \\
2.5 & 1.313626701 & 3.88773039 & 0.935 \\
2.6 & 1.325794066 & 3.91691483 & 0.930 \\
2.7 & 1.337326184 & 3.94435547 & 0.925 \\
2.8 & 1.348290809 & 3.97442102 & 0.921 \\
2.9 & 1.358745180 & 3.99858905 & 0.916 \\
3.0 & 1.368738107 & 4.02559814 & 0.912 \\
\hline
\end{tabular}

emergent solitary wave decreases. As $\gamma \downarrow \tilde{\gamma}$, the quantity $c^{*}$ appears to take longer to establish itself. It seems possible that, for fixed $c, c^{*}=c^{*}(\gamma)$ converges to $c_{p}$ as $\gamma \downarrow \tilde{\gamma}(c)$.

As an illustration of the foregoing, consideration is given to the long-time evolution of a solitary wave with initial speed $c=2.5$. For this case, the experimentally determined value of the amplitude perturbation threshold is $\tilde{\gamma}=0.935$. Figure 5 shows the evolution of the initial data $u_{0}=\gamma \phi_{c}$, for $c=2.5$ and $\gamma=0.94$. The long-time result shown here is typical of stable solitary waves $\phi_{c}$ when the amplitude-perturbation parameter $\gamma$ is above the critical value $\tilde{\gamma}(c)$. After a short period of adjustment, which will be elaborated presently, the solution is seen to feature a solitary wave of reduced total energy followed by a separated, dispersive tail. In contrast, Figure 6 shows the purely
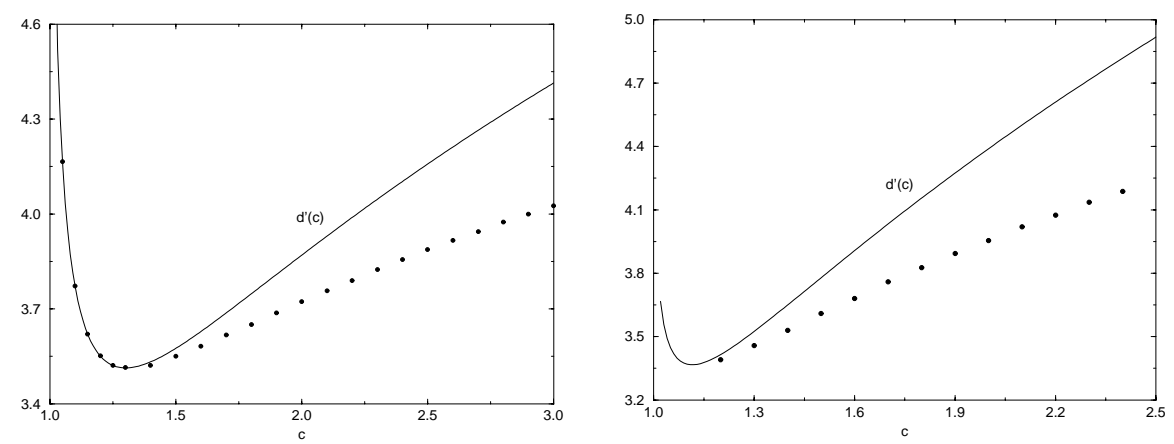

Fig. 4. The solid curve $d^{\prime}(c)$ and the points on $M_{\tilde{\gamma}}(c)$ for (a) $p=8$ and (b) $p=6$. 


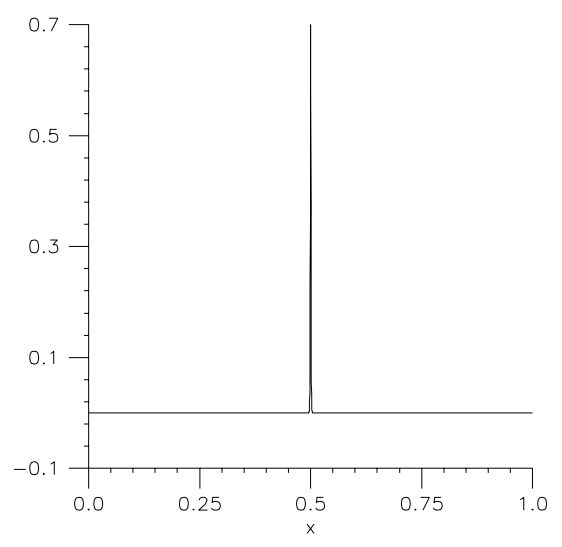

$\mathrm{t}=2.5$

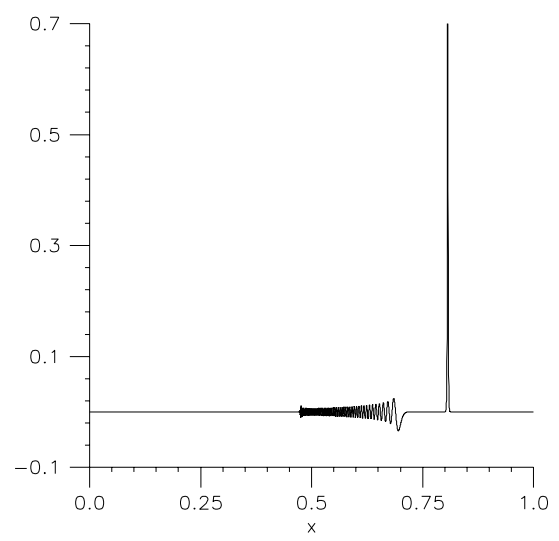

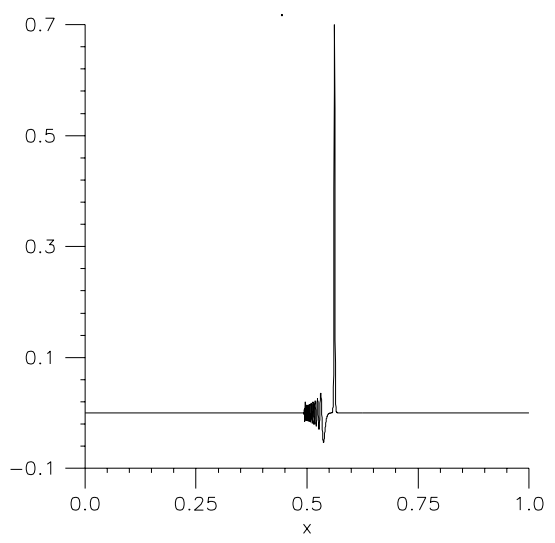

$t=5.0$

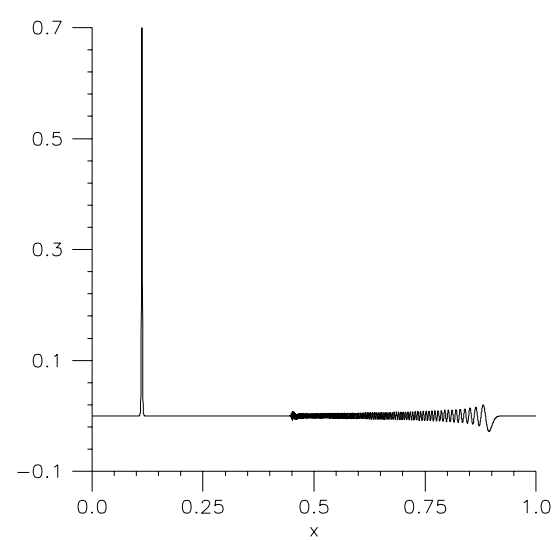

Fig. 5. Perturbed stable solitary wave with $c=2.5, \gamma=0.94$. The amplitude has been truncated in this illustration. At $t=5.00$, the sup-norm was approximately 1.12 .

dispersive waveform that evolves from $\gamma \phi_{c}$ with $c=2.5$ and $\gamma=0.93<\tilde{\gamma}(c)$. In Figure 5, the dispersive wave trailing the newly established, rightward-going solitary wave expands its support very slowly to the left while propagating to the right, and accompanied by a gradual decrease in overall amplitude, as expected from theoretical considerations (cf. [50], [51]). Similar behavior is noted for the entire solution depicted in Figure 6.

The adjustment period to which allusion was made earlier is discussed now. The behavior of the solution depicted in Figure 5 was typical of solutions emanating from $\gamma \phi_{c}$ when $\tilde{\gamma}(c)<\gamma<1$. Figure 7 shows more clearly how the amplitude and speed of the primary elevation of the solution in Figure 5 changed over time. Notice how quickly 


$$
\mathrm{t}=0.5
$$

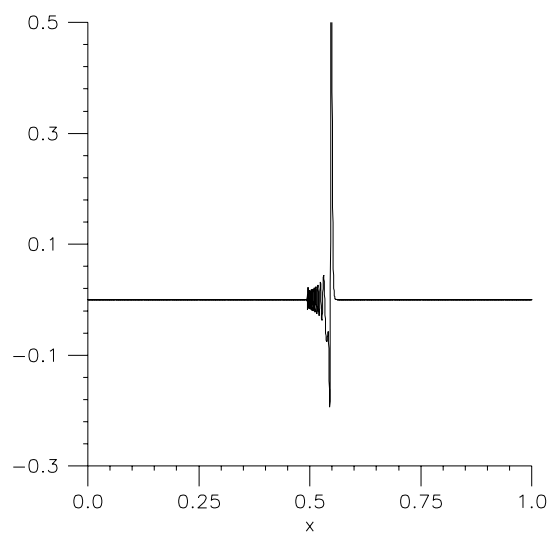

$\mathrm{t}=3.5$

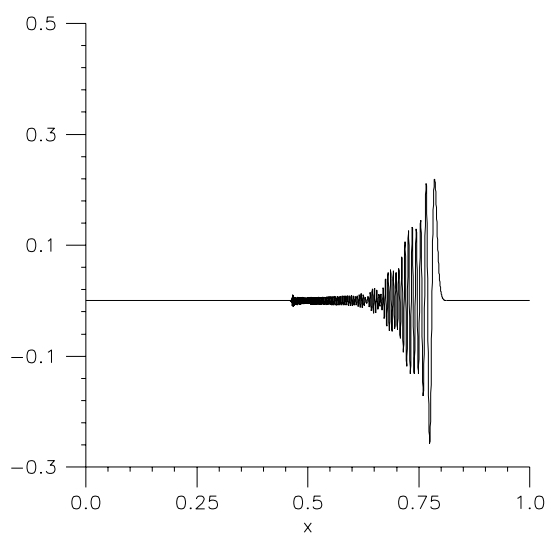

$\mathrm{t}=2.0$

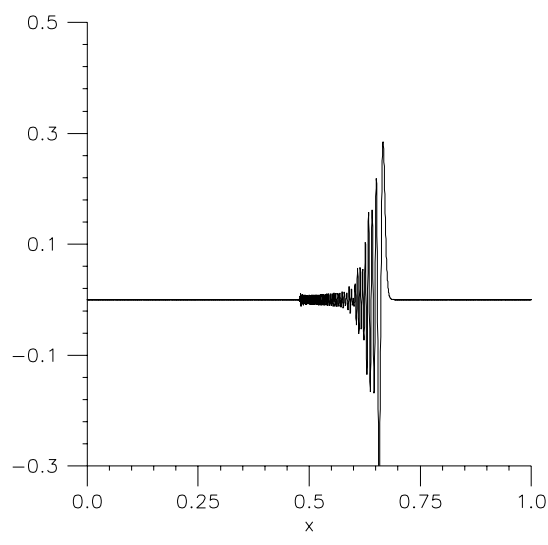

$t=5.0$

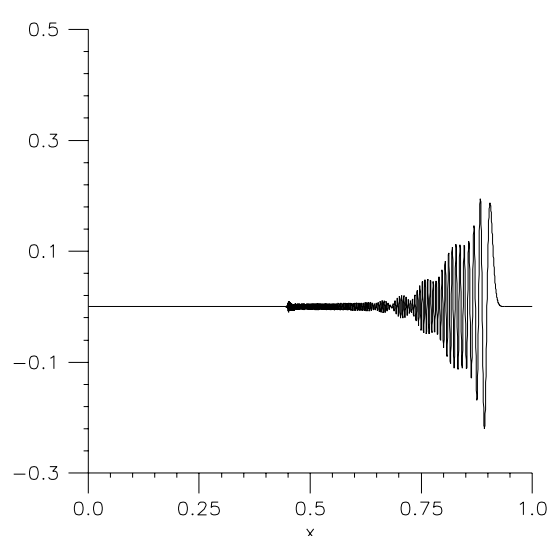

Fig. 6. Perturbed stable solitary wave with $c=2.5, \gamma=0.93$. Same initial data as in the previous figure. At $t=0.0$, the sup-norm was 1.21.

the primary elevation sheds energy and reconfigures itself as a new traveling wave. Not shown is a similar settling down of the shape of this elevation to $\phi_{c^{\star}}$, where $c^{\star} \approx 1.515$, as would be expected from either Figure 7 or Table 3 . Thus, the new solitary wave derived from the perturbed original solitary wave with $\gamma<1$ is slower, smaller, and broader than the original. (It is worth remark that, for $\gamma=1$, there is only a very small adjustment in speed and amplitude due to truncation and round-off error.) The case $\gamma>1$ will be highlighted below. A detailed view of the dispersive tail that appeared behind the emergent solitary wave $\phi_{c^{\star}}$ in Figure 5 is shown at $t=2.00$ in Figure 10.

On the other hand, for $\gamma<\tilde{\gamma}(c)$, Figures 6 and 8 capture the typical behavior of the process of loss of traveling-wave structure under large perturbations. The soliton-like 


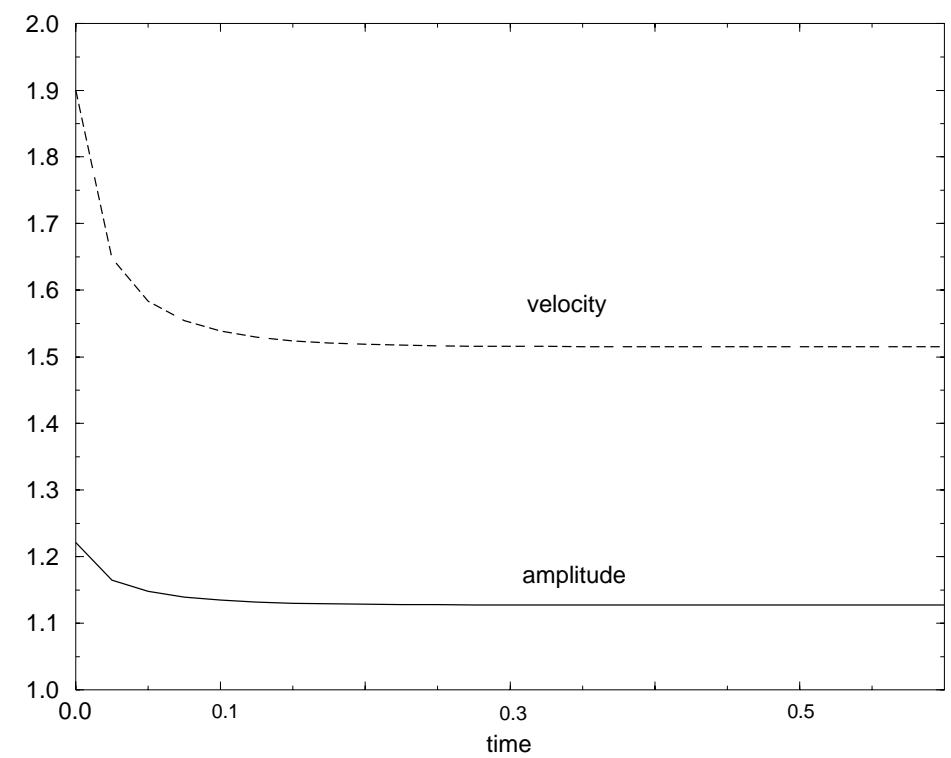

Fig. 7. Typical speed and amplitude time history for a perturbed stable solitary wave with $\gamma<1$. Initial speed and perturbation parameter were $c=2.5$, and $\gamma=0.94$, respectively.

solution loses its symmetry, the effect being more pronounced on the trailing edge of the solution's center. This is then followed by an increasingly prominent kink appearing at the back of the principal elevation, culminating in the collapse of the solution and an ensuing increase in support. If the collapsed solution and the dispersive tail interact, a beat pattern may be produced, as is the case in Figure 6. In Figure 8, two radiative tails are eventually all that is left after the instabilities overwhelm the perturbed solitary wave. One of the tails sheds immediately after the solitary-wave initial data is acted upon by the evolution equation in a manner that is familiar from the earlier simulations when $\gamma>\tilde{\gamma}(c)$. The second dispersive tail is the collapsed wave after the solution loses its single-humped form.

For $\gamma$ slightly below $\tilde{\gamma}$, it was found that the solution may take some time before it shows its asympotic form. Consider the results of an experiment in which a solution with the same parameters, i.e., $c=2.5$, but with $\gamma=0.934$, is integrated numerically. Figure 8 depicts the evolution of the solution for this case: The solution seems to develop into a solitary wave with speed $c^{*}=1.32$ at first, which would correspond to a stable case. However, it does not quite enter the region of attraction and eventually disperses away.

Stable solutions with $\gamma>1$ are quite different in their long-time behavior: Since the perturbation increases the solitary-wave initial data everywhere, slight perturbations can lead to considerable increase in amplitude. For example, a perturbation of $5 \%$ is capable of producing an amplitude increase in the resulting solution of $8 \%$ to $10 \%$. The solution apparently concentrates the extra mass in the center of the wave. With the increase in the amplitude there is an increase in the speed of the solitary wave and a narrowing of its width. Table 5 gives a summary of some of the experi- 


$$
t=1.0
$$

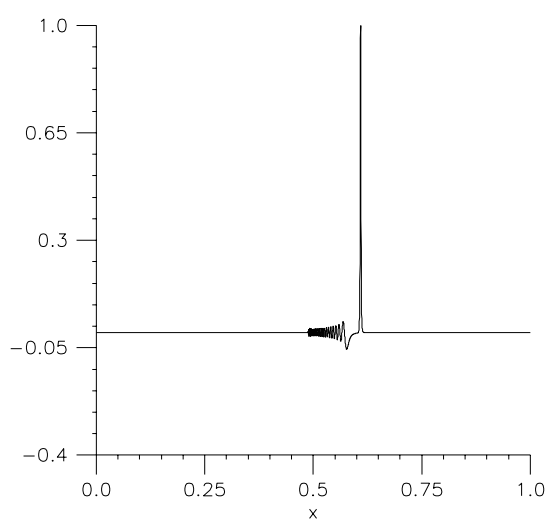

$\mathrm{t}=4.0$

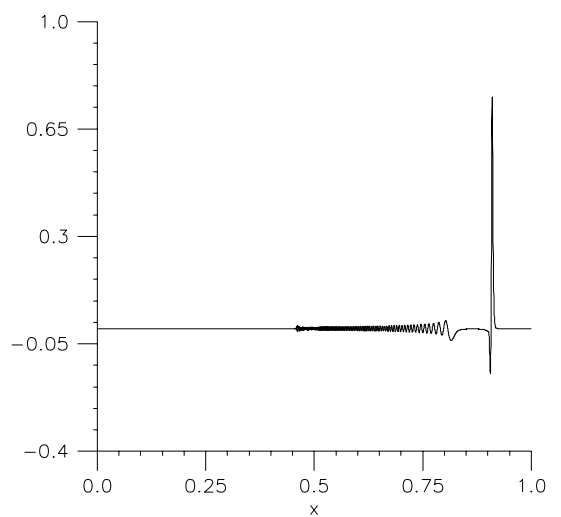

$\mathrm{t}=3.0$

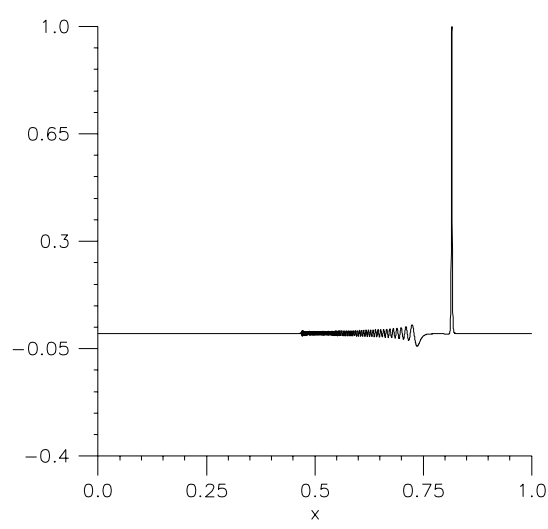

$\mathrm{t}=4.5$

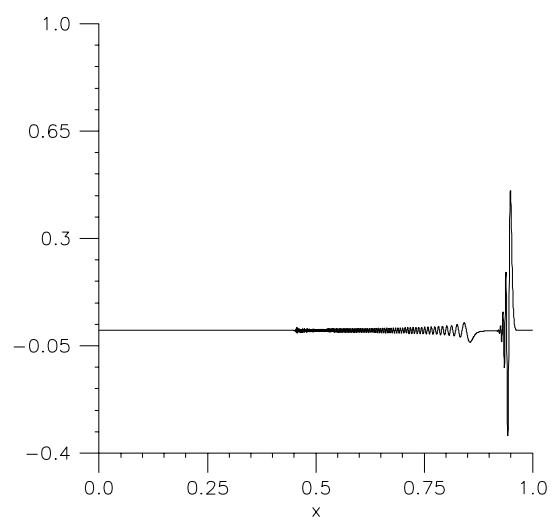

Fig. 8. Perturbed solitary wave with $c=2.5, \gamma=0.934$. The solution first resolves into a positive elevation followed by a dispersive tail; the elevation eventually collapses dispersively. At $t=0.0$, the sup-norm was 1.21 .

ments on stable solitary waves for various values of $\gamma$. The last two columns of the table provide information regarding the measured amplitude and computed speed of the wave at time $T=5.00$. Figure 9 shows the evolution of a typical case when $\gamma>1$. The main feature of the solution is the very small radiative tail produced. Comparison of Figure 10 with Figure 9, the former showing the long-time shape of a solitary wave with $c=2.5$, but with $\gamma=0.94$, shows definite shape differences in the solution.

To investigate the effect of perturbations in the width of stable solitary waves, initial data of the form

$$
U(x, 0)=A\left\{\operatorname{sech}^{2}[\lambda K(x-c t)]\right\}^{1 /(p-1)},
$$


Table 5. Evolution of perturbed stable solitary waves of speed $c$ and with the amplitudeperturbation parameter $\gamma>1$. Recorded is the speed $c^{*}$ and amplitude $A^{*}$ of the resulting solitary wave at time $T=5.00$.

\begin{tabular}{ccccc}
\hline$c$ & $A$ & $\gamma$ & $c^{*}$ & $A^{*}$ \\
\hline 1.500 & 1.123 & 1.010 & 1.591 & 1.150 \\
1.500 & 1.123 & 1.020 & 1.689 & 1.175 \\
2.000 & 1.240 & 1.020 & 2.144 & 1.264 \\
2.000 & 1.240 & 1.040 & 2.378 & 1.298 \\
2.000 & 1.240 & 1.060 & 2.630 & 1.329 \\
2.000 & 1.240 & 1.080 & 2.898 & 1.358 \\
2.500 & 1.314 & 1.020 & 2.623 & 1.328 \\
2.500 & 1.314 & 1.040 & 2.922 & 1.361 \\
\hline
\end{tabular}

where $A$ and $K$ are given by Eq. (6), were used as input in the experiments that follow. The initial data $U(x, 0)$ given above is recognized as the gRLW solitary-wave solution when $\lambda=1$. The real parameter $\lambda$ is used to change the width of the initial data and thus

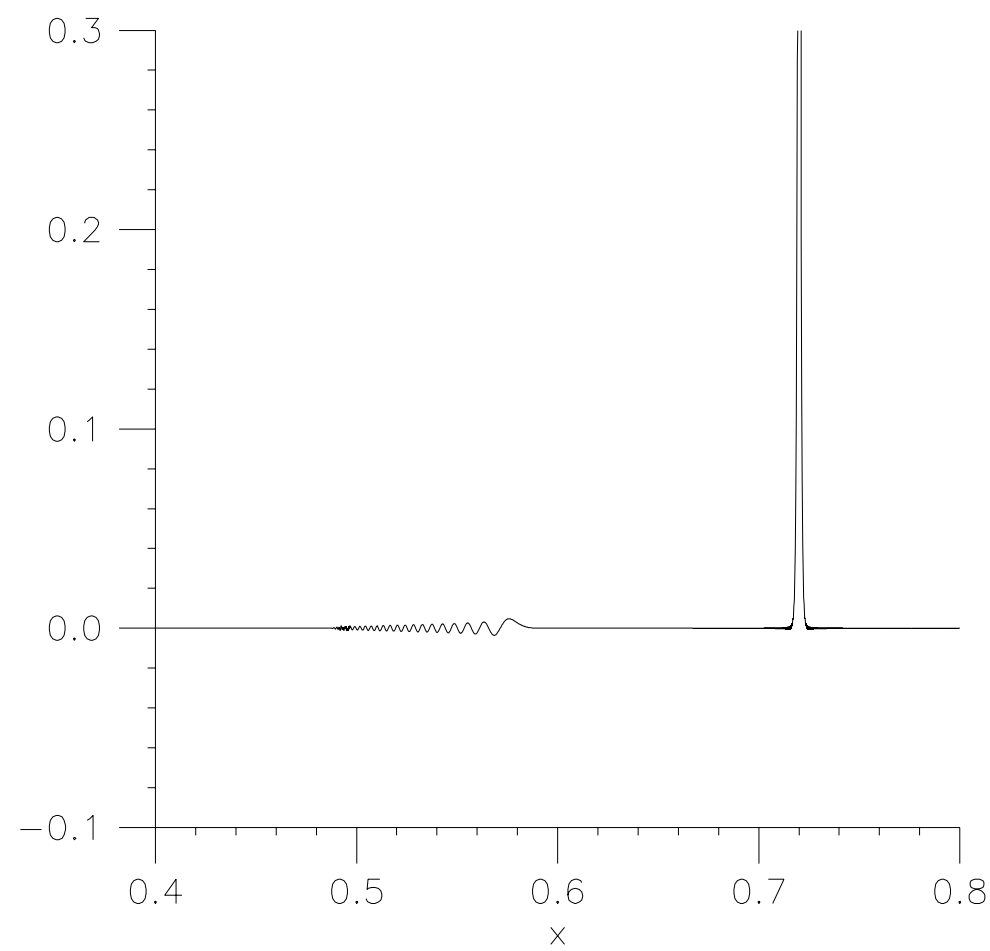

Fig. 9. Solitary wave at $t=1.00$ with $\gamma>1$. The peak of the solution has been truncated to show details of the dispersive tail. The initial speed was $c=2.5$ and $\gamma=1.02$. 


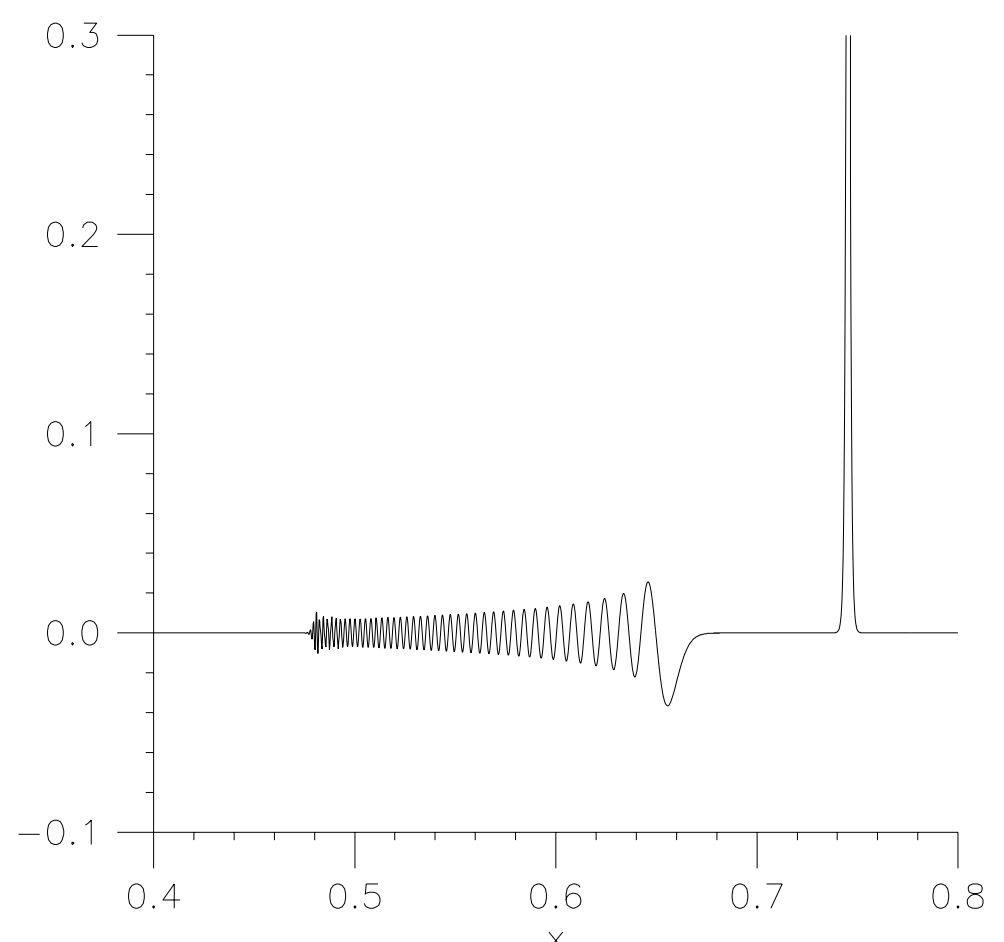

Fig. 10. Solitary wave at $t=2.00$ with perturbation $\gamma<1$. The initial speed was $c=2.5$ and $\gamma=0.94$. At $t=2.00$, the sup-norm was 1.128.

will be known henceforth as the width perturbation parameter. Guided by the results for amplitude perturbations, we expect that for some values of $\lambda$, the solution will retain a solitary wave in its long-time evolution, while for others, a purely dispersive asymptotics will be evident. Indeed, it turns out that for fixed $p$ and any $c>c_{p}$, there is a width demarcation $\tilde{\lambda}=\tilde{\lambda}(c)$ such that retention of a solitary wave in the temporal asymptotics is associated with $\lambda<\tilde{\lambda}$. It is worth noting that, for initial data $u(x, 0)=\phi_{c}(\lambda x)$, the resulting solution has the property

$$
\|u(\cdot, t)\|_{H^{1}}^{2}=\|u(\cdot, 0)\|_{H^{1}}^{2}=\lambda^{-1}\left\|\phi_{c}\right\|_{L_{2}}+\lambda\left\|\phi_{c}^{\prime}\right\|_{L_{2}} .
$$

Hence, the size of the $H^{1}$-norm of the initial data is not the determining factor for whether a perturbed solitary wave will ultimately completely disperse.

In more detail, it has been shown by Albert [50] that solutions of the initial-value problem (2) that begin with sufficiently small norm disperse asymptotically as $t \rightarrow \infty$ if $p>5$. The present simulations indicate that positive initial data with a large $H^{1}$-norm may also disperse completely. This contrasts with the KdV equation (and perhaps the RLW equation), where it seems, for example, that any positive initial data leads to a solution that features at least one solitary wave in its long-time asymptotics.

The numerical results reported in Table 6 give precision to the above commentary. For $\lambda$ below $\tilde{\lambda}=\tilde{\lambda}(c)$ an emergent solitary wave is clearly identifiable, whereas for $\lambda$ larger 
Table 6. Speed $c$ and approximate value of the width perturbation $\lambda$ that defines the transition between purely dispersive and nondispersive regimes. The outcome is dispersive for $\lambda>\lambda^{*}$, the numerically determined approximation of $\tilde{\lambda}$. Here, $p=8$.

\begin{tabular}{cc}
\hline$c$ & $\lambda^{*}$ \\
\hline 1.00100 & 1.00 \\
1.30092 & 1.00 \\
2.00000 & 1.11 \\
2.50000 & 1.15 \\
3.00000 & 1.19 \\
\hline
\end{tabular}

than $\tilde{\lambda}$, complete dispersion was observed. The range of $\lambda$ over which transitions from dispersive to solitary-wave outcomes was systematically investigated by performing a series of experiments in which the value of $\lambda$ was changed in increments of $1 \%$. The length of integration in the experiments was several times longer than was necessary to determine the long-term asymptotics of a particular initial datum. Some of the resulting estimates of $\lambda^{*}$ are reported in Table 6.

\section{Experiments with Unstable Solitary Waves}

In this section, the evolution of solutions emanating from initial data which are perturbations of exact analytical solutions of Eq. (2) with $p>5$ and with $1<c \leq c_{p}$ are featured. Such data corresponds to unstable solitary-wave solutions of the gRLW equation (see the stability curve for $p=8$ illustrated in Figure 2). The perturbation parameter $\gamma$ is used once more to effect an amplitude perturbation. Qualitatively, the long-time evolution of perturbations of unstable solitary-wave solutions may be characterized by two types of behavior, depending on the value of $\gamma$. When $\gamma \geq 1$, the solution emanating from the initial data will resolve itself into one or more solitary waves, sometimes accompanied by additional structure. On the other hand, the same initial data but with $\gamma<1$ will inevitably disperse in the course of its evolution. The dispersive outcome is similar to that observed in Section 3 for amplitude-perturbations of stable solitary waves when $\gamma<\tilde{\gamma}(c)$. Another way of expressing this is the specification $\tilde{\gamma}(c)=1$ when $1<c<c_{p}$ and $p>5$. In the rest of this study, attention will be given to evolutions starting with $\gamma \phi_{c}$, where $\gamma>1$.

All experiments reported in this section were carried out using $p=8$, and $\alpha=\beta=1$. The choices for the discretization remain the same as those taken in the previous section. The choice of values of $c$ and $\gamma$ in the experiments deserves further comment. Since unstable solutions have a speed $c$ in the range $1<c \leq c_{p}$ and since $c_{p}$ is near to 1 , the waveforms $\phi_{c}$ in question possess small amplitudes and large width. With regard to conducting numerical experiments with this type of data, the relative distribution of mass and energy of the solutions makes it imperative that integrations be performed over 


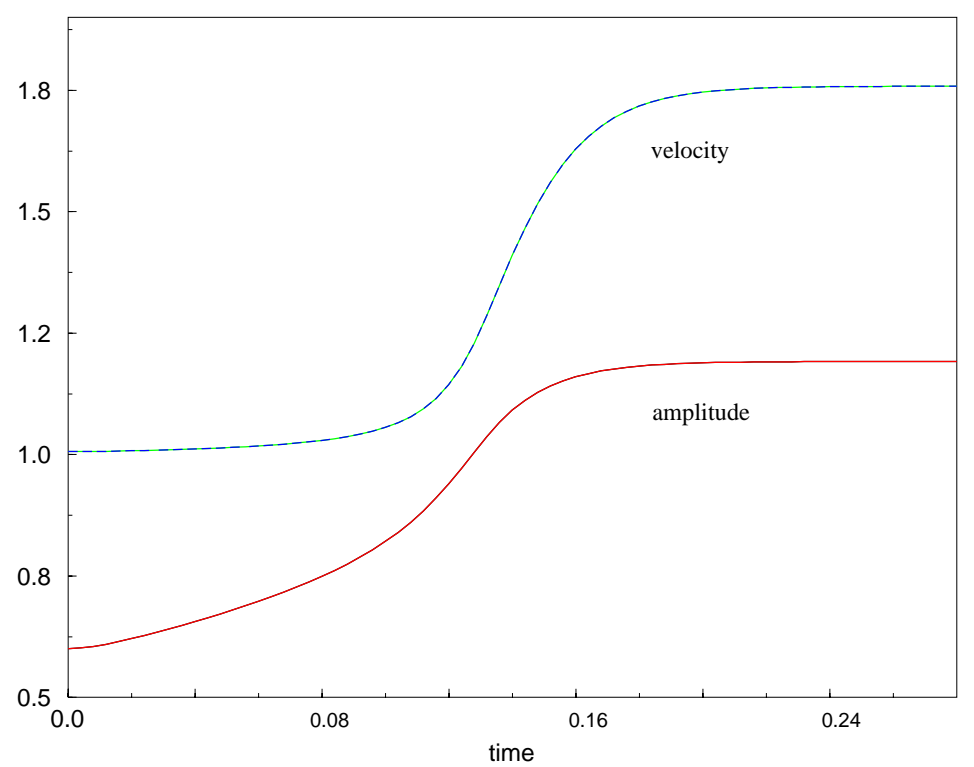

Fig. 11. Evolution of the speed and maximum value typical of the unstable solutions, as a function of time. Unstable perturbed solitary wave with $c=1.001, A=0.601 ; \gamma=1.30$. The asymptotic speed is $c^{*}=1.759$ and the long-time value of $\mathrm{A}$ is $A^{*}=1.192$.

long temporal and spatial intervals. This, in turn, poses a computational challenge, not only with regard to computing resources, but more importantly, in the preparation of the initial data. The way in which we achieved control of the support of the data is through the use of the scaling parameter mentioned earlier, which in the experiments reported in this section was set to 0.0004 . This scaling parameter concentrates the support of the initial data and mitigates problems with wrap-around of the solution and the eventual interaction of the solution with itself. If the solution is integrated long enough, the interaction is unavoidable; hence, careful choices of the scaling parameter, and of $c$ and $\gamma$, were made so that the solution could be integrated long enough to make an assessment of the likely asymptotics of these unstable solitary-wave initial data. To keep the number of time steps reasonable and, at the same time, resolve the long-time dynamics, initial data was chosen with a high energy level, which in turn dramatically reduced the total time interval required to observe the details of the evolution of unstable solitary waves. Large energy values were achieved by choosing values of the speed close to, but larger than, 1 and by increasing $\gamma$.

The main feature of the evolution of unstable solitary-waves to an amplitude perturbation $\gamma>1$ is characterized by the eventual transition to a stable regime. Figure 11 illustrates the sup-norm and the speed of the solution as a function of time corresponding to a solution with $\gamma>1$. The speed shows a gradual increase in value initially, followed by a period of much more rapid, but still smooth, increase, after which it settles down to an asymptotic value as the wave reaches a stable configuration. In this figure, an unstable exact solitary wave with parameters $c=1.001$ and amplitude-perturbation parameter $\gamma=1.30$ was used as initial data. 
Three types of long-time outcomes for perturbed unstable solitary-wave solutions are reported in this section. The first experiment features a solution which, as a consequence of its initial data, makes the transition to a stable regime, producing a highly structured dispersive tail in the process. In the second experiment, an unstable solitary-wave solution will be shown to make the transition to the stable regime, shedding some of its mass to form a secondary hump that shows little further amplitude change over long time intervals. The third experiment shows a solitary wave making the transition to the stable regime, producing a second stable solitary-wave in the process.

In the first experiment, a slightly perturbed exact solitary-wave was used as initial data: In terms of Eq. (20), the parameters were $\gamma=1.03$, and $c=1.001$. The resulting evolution is presented in Figures 12 and 13. The effects of the perturbation on the solution are clearly evident in the loss of symmetry, followed by the appearance of the dispersive tail; in the process the amplitude grows considerably. Sometime in the interval $t=1.28$ to $t=1.92$ the solitary wave reaches a stable configuration, remaining unchanged in amplitude and speed, and in the process, a well-defined dispersive tail develops. The speed and amplitude settle down to an estimated $c^{*}=1.71$ and $A^{*}=1.18$, respectively. An analytical solitary wave was subtracted from the solution at $t=3.00$, using the procedure described in Section 2, and the last frame in Figure 12 shows the dispersive tail by itself. Figure 13 shows the details of the solution minus the solitary wave at $t=9.00$. The tail eventually shows complete dispersion, in general agreement with linear theory (see again [50], [51]).

Figures 14 and 15 illustrate another outcome for a solution originating from the same initial conditions as above, but with $c=1.001$ and $\gamma=1.20$. In this case, the solitary wave quickly speeds up and develops additional structure. The structure starts becoming evident on the trailing edge of the wave by $t=0.16$. At $t=0.32$, a secondary structure separated from the leading wave is evident, and the leading solitary wave stabilizes with a constant speed and amplitude. In these figures, the solution shown has wrapped around in the intervening time, sometime between $t=0.32$ and $t=0.80$. A subtraction of the leading wave was performed on the numerical solution at $t=0.80$ by the procedure described heretofore. Hence, at later times, the plots only portray the evolution of the secondary structure plus the dispersive tail. The full solution is composed of the stable solitary-wave plus the hump featured in the remaining plots corresponding to this experiment. As seen in the figures, the secondary structure is not quite symmetric about its center of mass, so it is not itself a traveling wave (recall that the only bounded traveling waves tending to zero at $\pm \infty$ are those defined by (6), or minus those in case $p$ is odd). Close examination of the solution revealed that the hump has a speed and amplitude that are constant to six decimal places for a very long time. The sup-norm data showed that the solution did decay, albeit extremely slowly. Hence, it does not appear to be a simple dispersive tail or a solitary wave. Presumably, this structure will eventually form part of the dispersive tail, but we did not come to a firm conclusion.

If the initial energy is large enough, it is possible to generate a solution that resolves into a collection of solitary waves. In the last experiment described here, a perturbed unstable solitary wave is shown reaching the stable regime by shedding a second stable solitary-wave. To achieve such an outcome, the initial unstable solitary data had to be given enough energy to produce two stable solitary waves. Figures 16 and 17 illustrate how such a process occurred in the experiment. The initial solitary-wave of speed 


$$
\mathrm{t}=0.0
$$

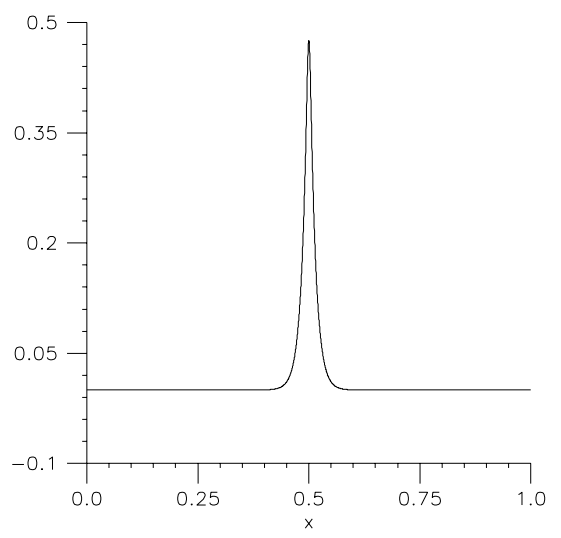

$\mathrm{t}=3.0$

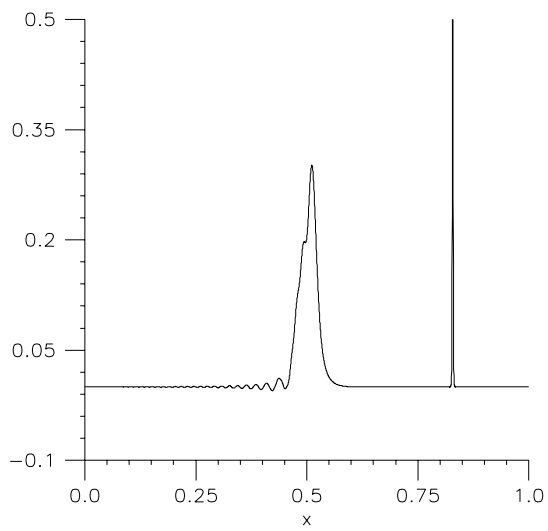

$t=2.4$

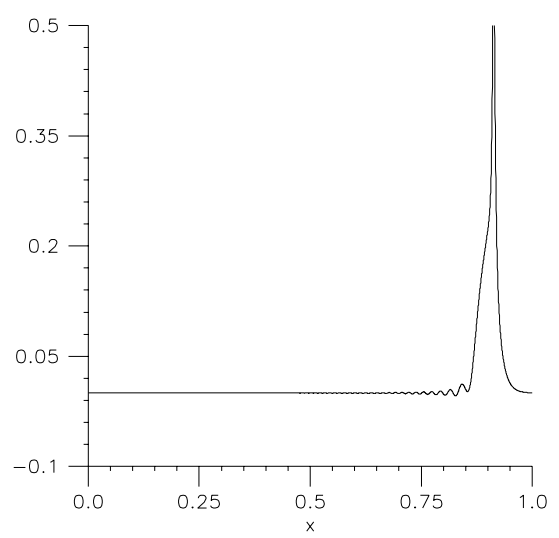

$t=6.0$

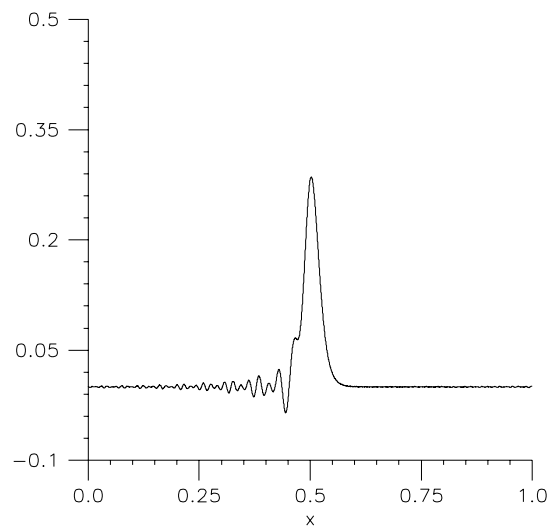

Fig. 12. Unstable perturbed solitary wave with $c=1.001$ and $\gamma=1.03$. At $t=3.00$ the supnorm was 1.144 . Subtraction of the of the leading solitary wave took place at $t=3.00$. The actual solution is composed of a solitary wave and a large-amplitude dispersive tail.

$c=1.001$ was given an amplitude perturbation of $\gamma=1.30$. With these values of the parameters, it was calculated that should a first solitary wave establish itself in the solution, there could still be enough energy in the remainder of the solution to permit a second stable solitary-wave to emerge. This outcome is shown in the figures, and it occurs around $t=0.20$. As was expected, the slower waves are more distant from the manifold of stable solutions, so that the transition to stable solutions takes longer. Figure 11 shows a typical increase in speed and amplitude of the solution as it makes its transition into a stable configuration. In the experiment featured in Figures 16 and 17, the subtraction of the leading solitary wave was carried out at $t=0.64$. As seen in the figures, for $t>0.64$, what is left behind in the solution is a hump that loses symmetry and 


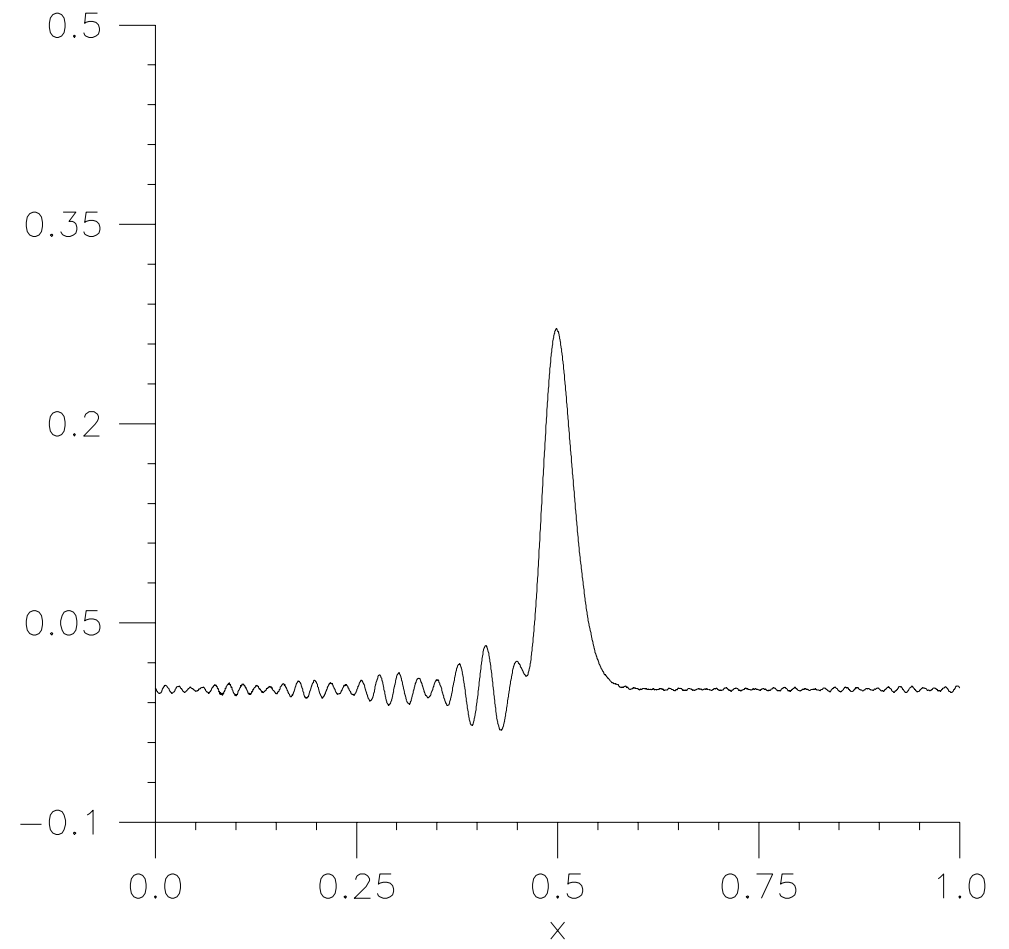

Fig. 13. Unstable perturbed solitary wave at $t=9.00$, with $c=1.001$ and $\gamma=1.03$. Solution shown after a solitary wave has been subtracted from the computed solution. Subtraction of the solitary wave took place at $t=3.00$.

develops a radiative tail. Eventually, the hump resolves into a second solitary-wave and a dispersive tail. The plots show the solution with the leading solitary wave subtracted. The secondary solitary-wave was found to possess constant amplitude and speed to 11 decimal places over the remainder of the integration time.

\section{Conclusions}

As was established in [42], solitary-wave solutions of Eq. (2) are orbitally stable for $p \leq 4$. For $p>5$, they are orbitally stable if their speed exceeds a critical level $c_{p}$. Otherwise, they are unstable. The present study was initiated by asking what happens to an unstable solitary wave when it is perturbed in an unstable direction. The extant theory is not helpful to answer this query, and consequently numerical techniques were developed to explore the dynamics of solutions to the generalized Regularized Long-Wave equation initialized with various perturbations of exact stable and unstable solitary-wave solutions.

To explore the dynamics of the gRLW equation (2) in a neighborhood of its solitarywave solutions, $\left\{\phi_{c}\right\}_{c>1}$, amplitude and width perturbations were proposed of the form

$$
u(x, 0)=\gamma \phi_{c}(\lambda x)
$$




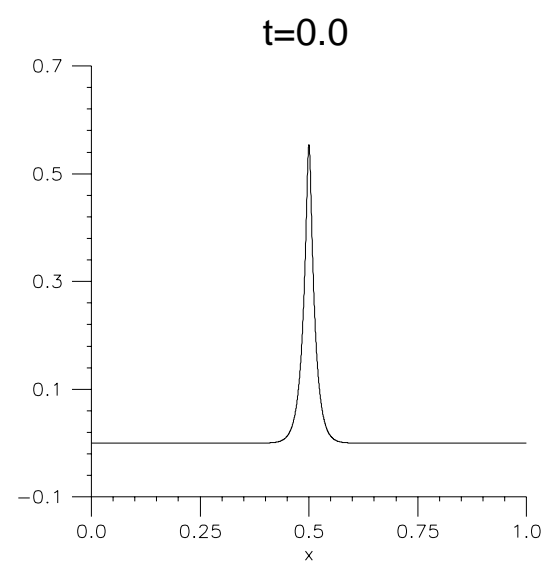

$t=3.2$

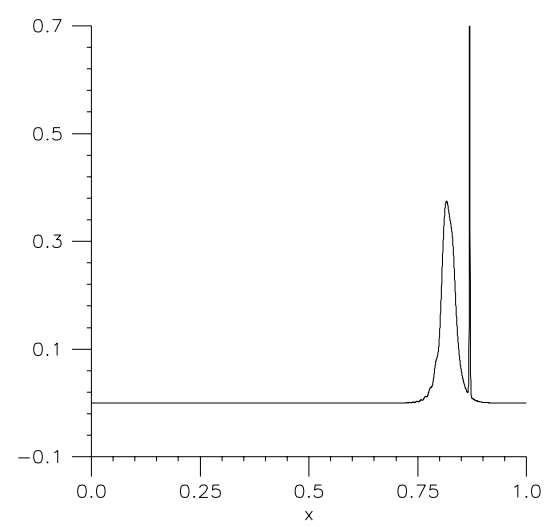

$\mathrm{t}=1.6$

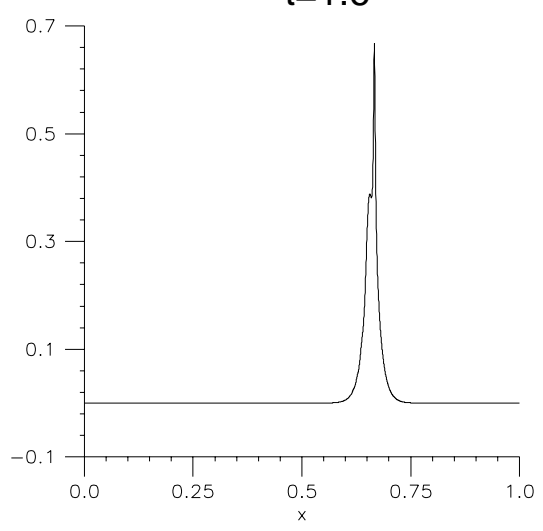

$t=8.0$

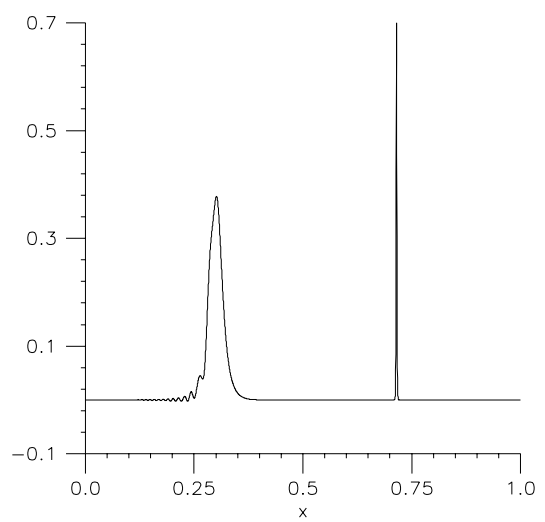

Fig. 14. Unstable perturbed solitary wave, $c=1.001$ and $\gamma=1.20$. Subtraction of leading solitary wave took place at $t=0.80$. The actual solution for $t>0.80$ composed of a solitary wave and a large trailing bump.

where $\phi_{c}$ is as in (6). If both $\gamma=\lambda=1$, the solution is the traveling wave $u(x, t)=$ $\phi_{c}(x-c t)$, and interest was focused on what happens when $\gamma$ or $\lambda$ is not equal to 1 . Although not explicitly reported here, numerical experiments performed with $\lambda=\gamma=1$ were in accordance with the theory of Souganidis and Strauss on stability of solitary-wave solutions of the generalized Regularized Long-Wave equation.

In the course of our study, we have come to definite views about what happens to the perturbed stable and unstable solitary waves, but in addition, we have formed a tentative picture of the long-time asymptotics of solutions corresponding to more general initial data.

The outcome of perturbing a stable solitary wave is just what is expected if the perturbation is small, and corresponds to what Miller and Weinstein [41] proved for the case $p=4$. The evolution causes the solution to lose a little mass in the form of a 

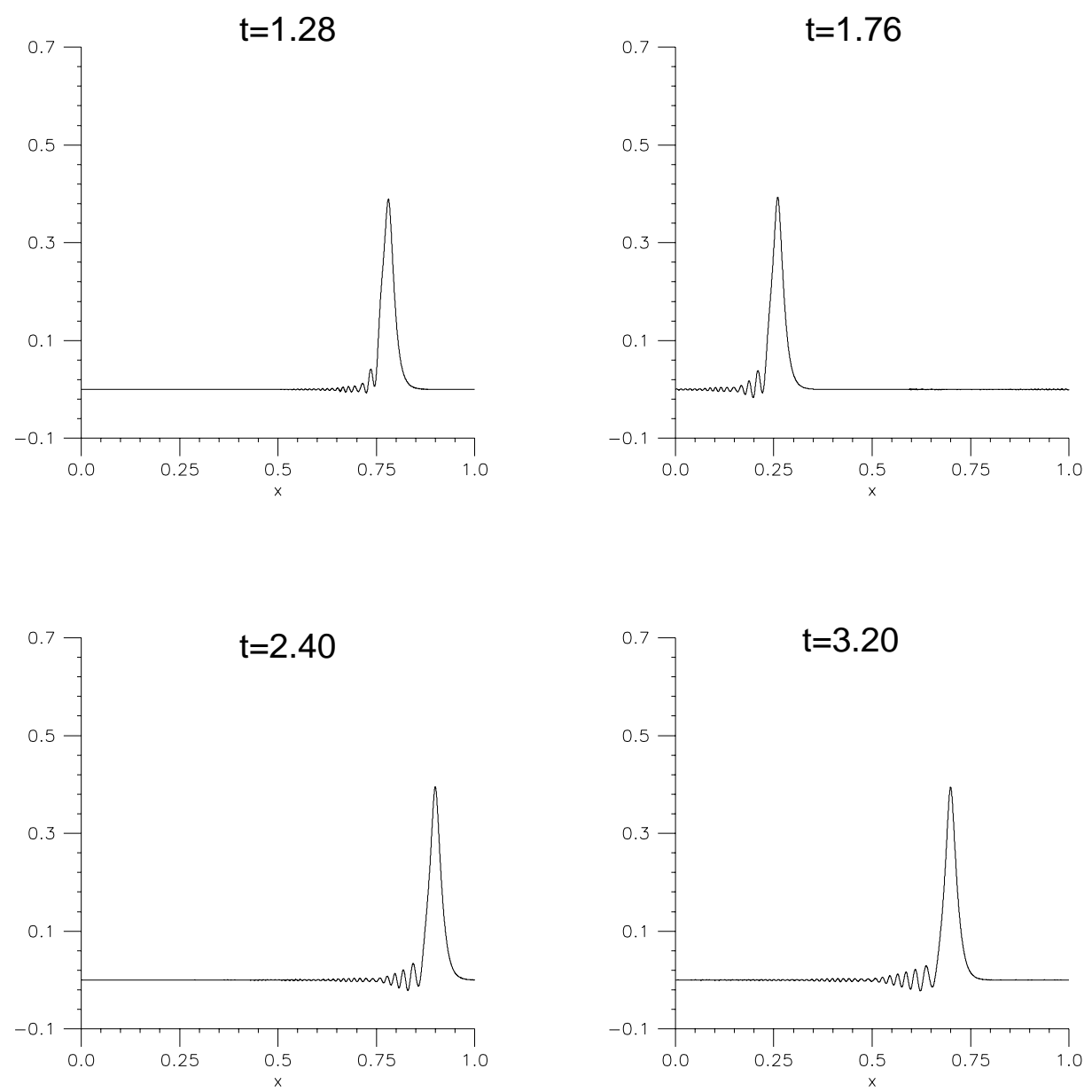

Fig. 15. Unstable perturbed solitary wave with $c=1.001$ and $\gamma=1.20$. At $t=0.80$, the sup-norm was 1.183 . Subtraction of the leading solitary wave took place at $t=0.80$. The actual solution for $t>0.80$ was composed of a solitary wave and a large trailing bump.

dispersive tail, with the bulk converging rapidly to a nearby stable solitary wave. On the other hand, we found that larger perturbations can push the solution out of the range of attraction of the solitary waves altogether, and into a state where dispersion dominates despite reasonably large amplitudes.

The outcome of perturbing an unstable solitary wave, even for small perturbations, is more interesting. One possibility which does manifest itself is that the wave gives over to large-amplitude dispersion, like that which obtained for a certain class of substantial perturbations of stable solitary waves. The other thing that happens is that the wave decomposes into one or more stable solitary waves followed by a dispersive tail.

These simulations together with the certitude that the solitary-wave solutions are the central ingredient in the long-time asymptotics lead to the following expectation. A general disturbance will resolve into a sequence of solitary waves in the stable range, 
$t=0.00$

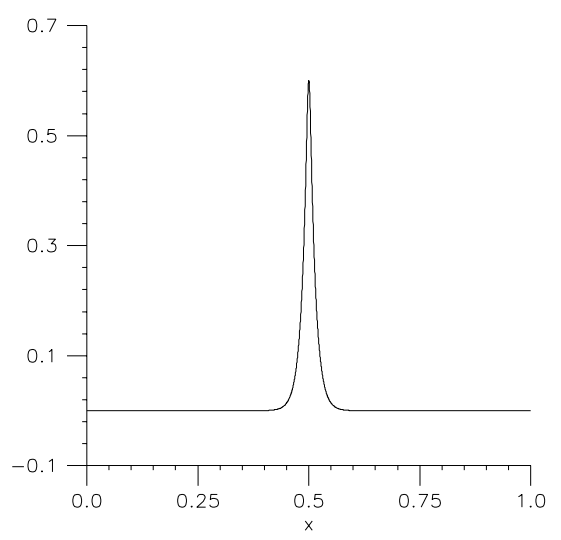

$t=0.16$

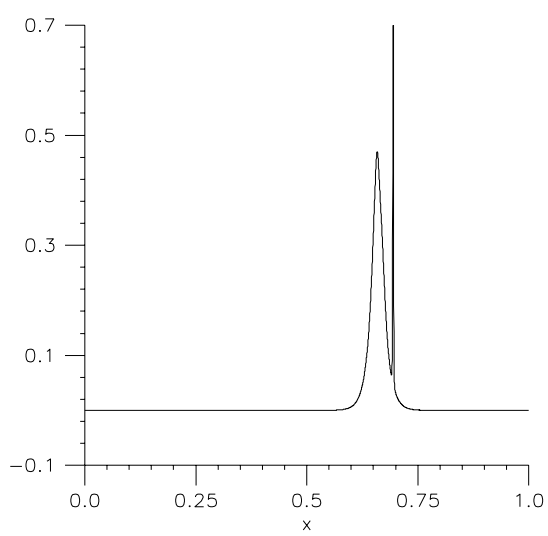

$t=0.06$

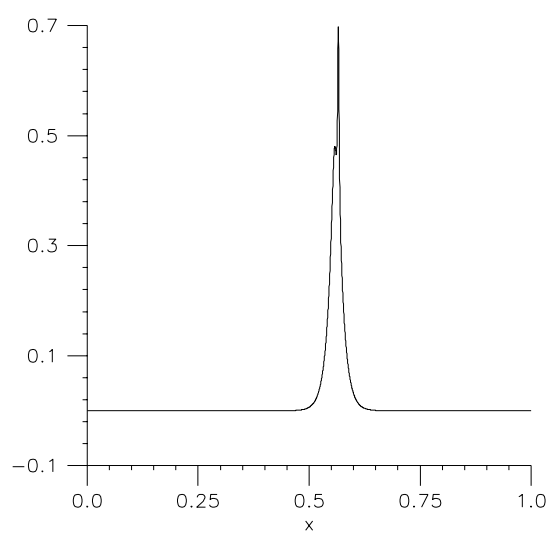

$t=0.64$

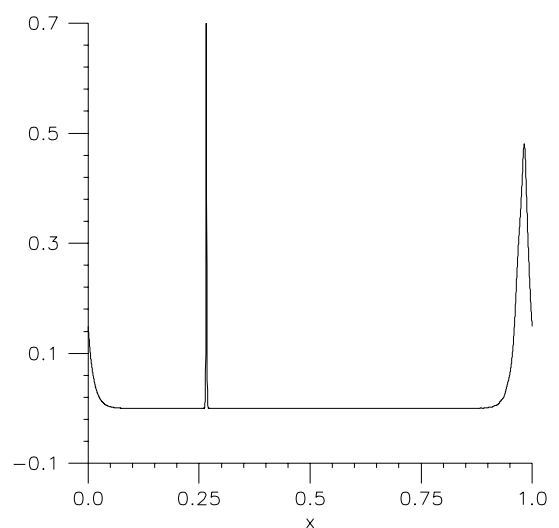

Fig. 16. Evolution of an unstable perturbed solitary wave with $c=1.001$ and $\gamma=1.30$. At $t=0.16$, the sup-norm is 1.141 , and at $t=0.64$ it is 1.16458 . Subtraction of the leading solitary wave took place at $t=0.64$. For $t>0.64$, the solution was composed of two solitary waves and a dispersive tail.

ordered by amplitude with the larger waves in the front, followed by a dispersive tail which need not be of small amplitude, but which spreads and decreases slowly, as is the wont of such structures.

A standard test case for this type of conjecture is to initiate the wave motion with a Gaussian pulse. Consider the case $\alpha=\beta=1$ with initial data

$$
U(x, 0)=0.63 \exp \left(-0.0125(x-0.4)^{2}\right)
$$

and various values of $p$. Because of its very rapid decrease away from its crest, it is straightforward to consider this as periodic input, just as for the perturbed solitary waves discussed earlier. 


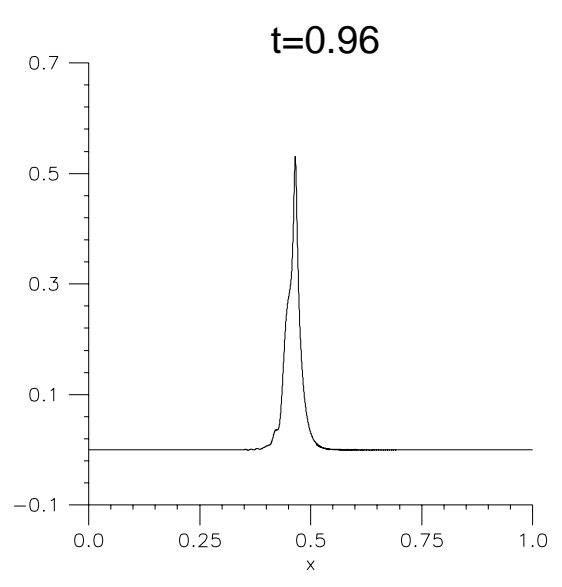

$t=1.60$

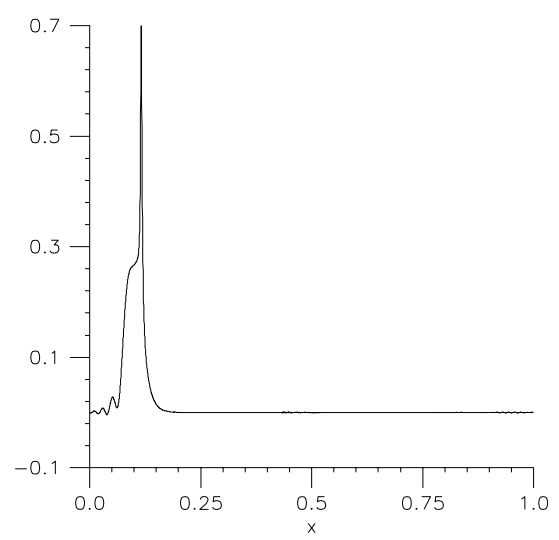

$t=1.28$

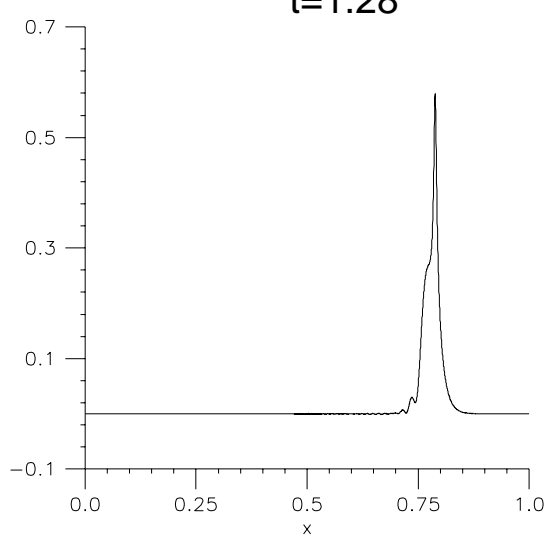

$\mathrm{t}=1.92$

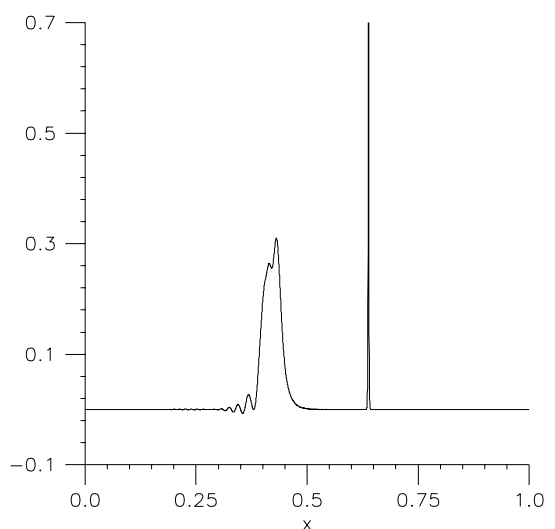

Fig. 17. Evolution of an unstable perturbed solitary wave with $c=1.001$ and $\gamma=1.30$. The sup-norm at $t=1.60$ is 0.754 , and at $t=1.92$ is 1.179 . Subtraction of the leading solitary wave took place at $t=0.64$. The actual solution after $t>0.64$ is composed of two solitary waves and a dispersive tail.

The evolution of an initial Gaussian pulse serves to illustrate this phenomenon. Consider the solution of (2) with $\alpha=\beta=1$ and $p=7$ or 8 , corresponding to initial data as in (23). Figure $18 \mathrm{~b}$ illustrates the case $p=8$ at the same times.

Figure 18 shows the initial stages of the evolution of this Gaussian profile for $p=7$ and $p=8$. The computational parameters related to this simulation are the same as those pertaining to Figures $8-10$, except that the spatial scaling is now $x \rightarrow 0.001 x$. Figure 18a shows three snapshots of the solution for $p=7$ while later stages of the evolution corresponding to $p=7$ are shown in Figure 19, where we have availed ourselves of the subtraction algorithm to remove isolated solitary-wave structures from the solution, after which the modified solution is allowed to evolve further. This process 


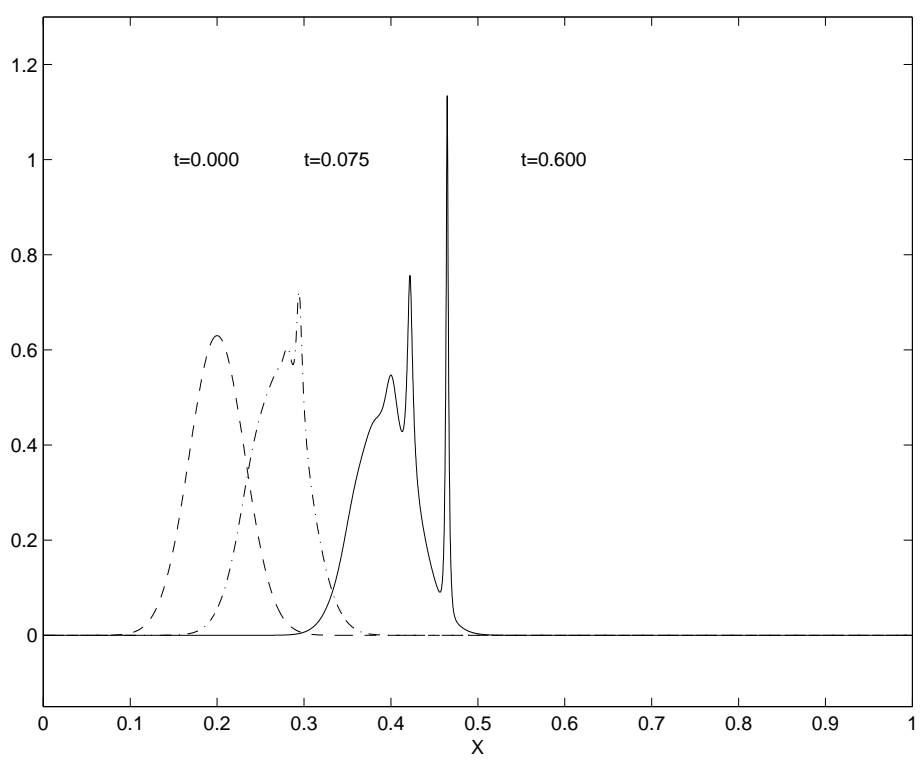

(a)

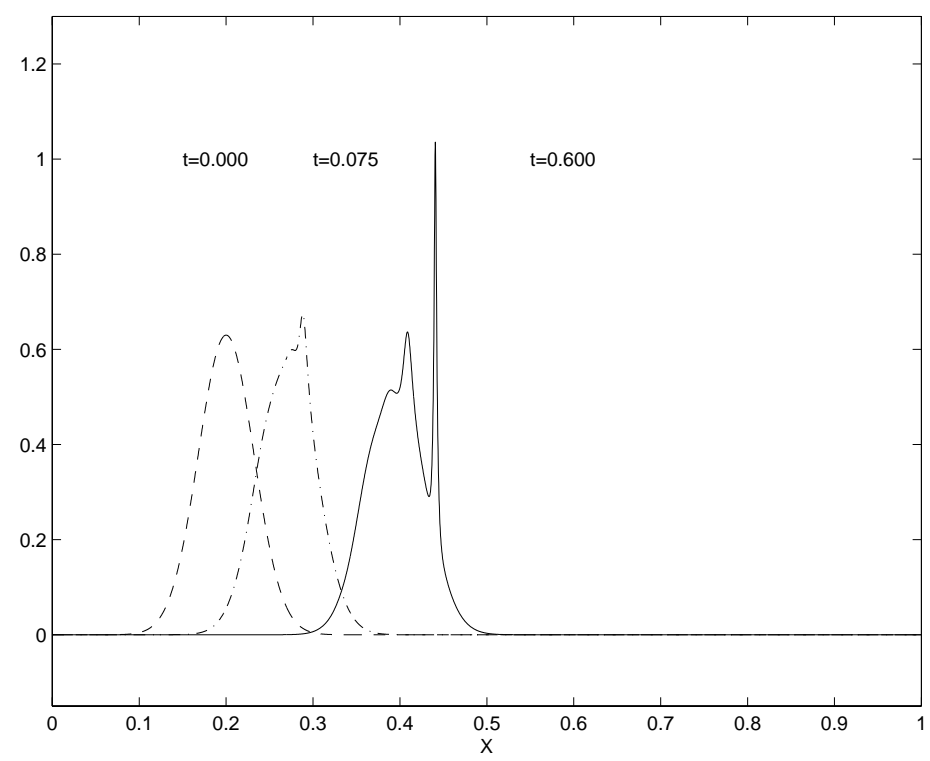

(b)

Fig. 18. Early stages of the evolution of Gaussian pulse, (a) $p=7$, (b) $p=8$.

is continued until the solution shows no evidence of further solitary-wave formation. The same process is illustrated in Figure $18 \mathrm{~b}$ and Figure 20, corresponding to $p=8$. The two solitary waves shown in Figure 20a are removed, and the calculation allowed 


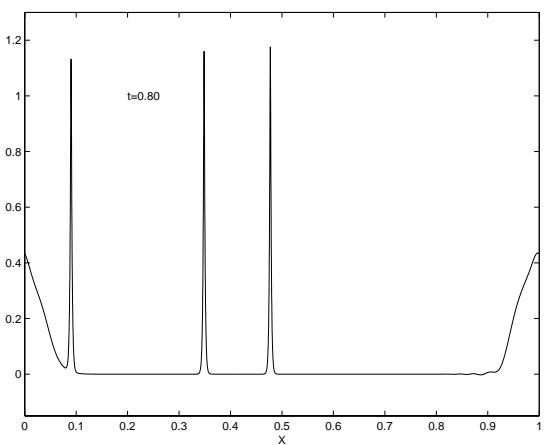

(a)

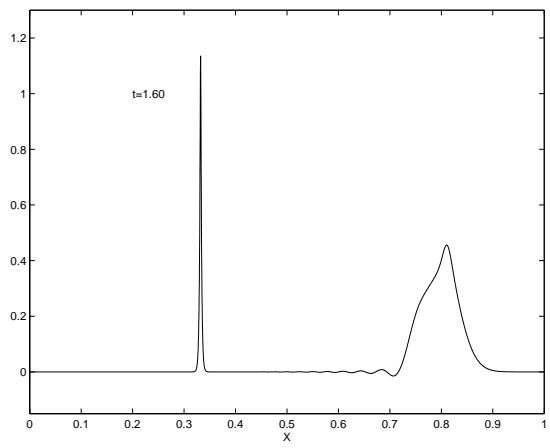

(c)

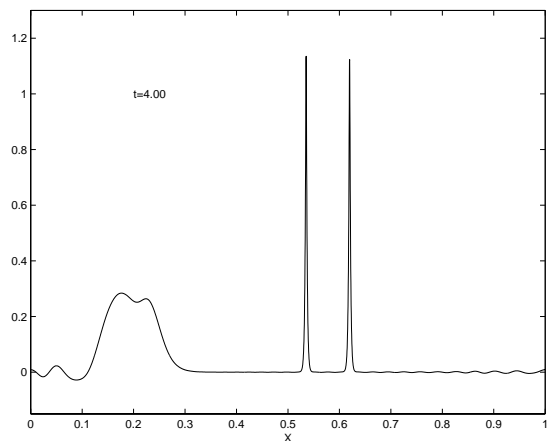

(b)

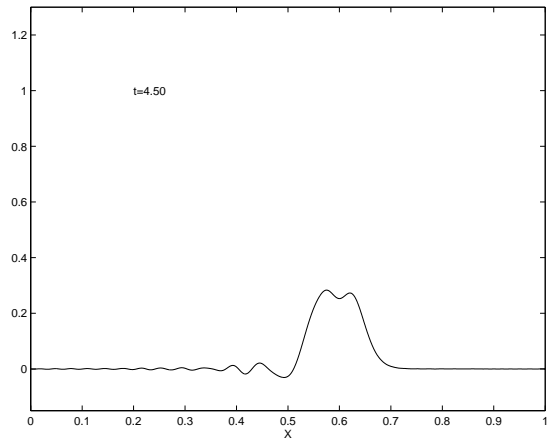

(d)

Fig. 19. Evolution of a Gaussian pulse, $p=7$. (a) At $t=0.80$. (b) At $t=1.60$ after the two isolated solitary waves shown in (a) have been subtracted. (c) At $t=4.0$ after the isolated solitary wave appearing in (b) has been subtracted. (d) At $t=4.5$ after the two isolated solitary waves appearing in (c) have been subtracted.

to continue. At $t=1.6$, a third solitary wave is about to separate from the bulk of the solution. A short time later, the separated solitary wave is removed, and at times greater than $t=4.0$, the solution showed no evidence of further solitary-wave resolution. However, the dispersive tail continues to evolve, spreading throughout the domain while losing overall amplitude.

Figure 21 shows the evolution of the initial data in (23) when $p=6$. The evolution is shown first in Figure 21a at $t=0.8$, where three solitary waves have broken away from the initial heap in exactly the way depicted in Figure 18 for $p=7,8$. These waves were subtracted shortly after $t=0.8$ via the algorithm described earlier. Figure $21 \mathrm{~b}$ shows further evolution to $t=2.25$ of the portion of the solution remaining after the three solitary waves are expunged. Note that a fourth solitary wave, already appearing in Figure 21a, has separated. Because of periodicity, it seems to be trailing the remainer. There is clear evidence of more solitary waves developing in the structure that remains. This run is particularly interesting because the solitary waves make their appearance in temporally separate phases rather than in a tight sequence. 

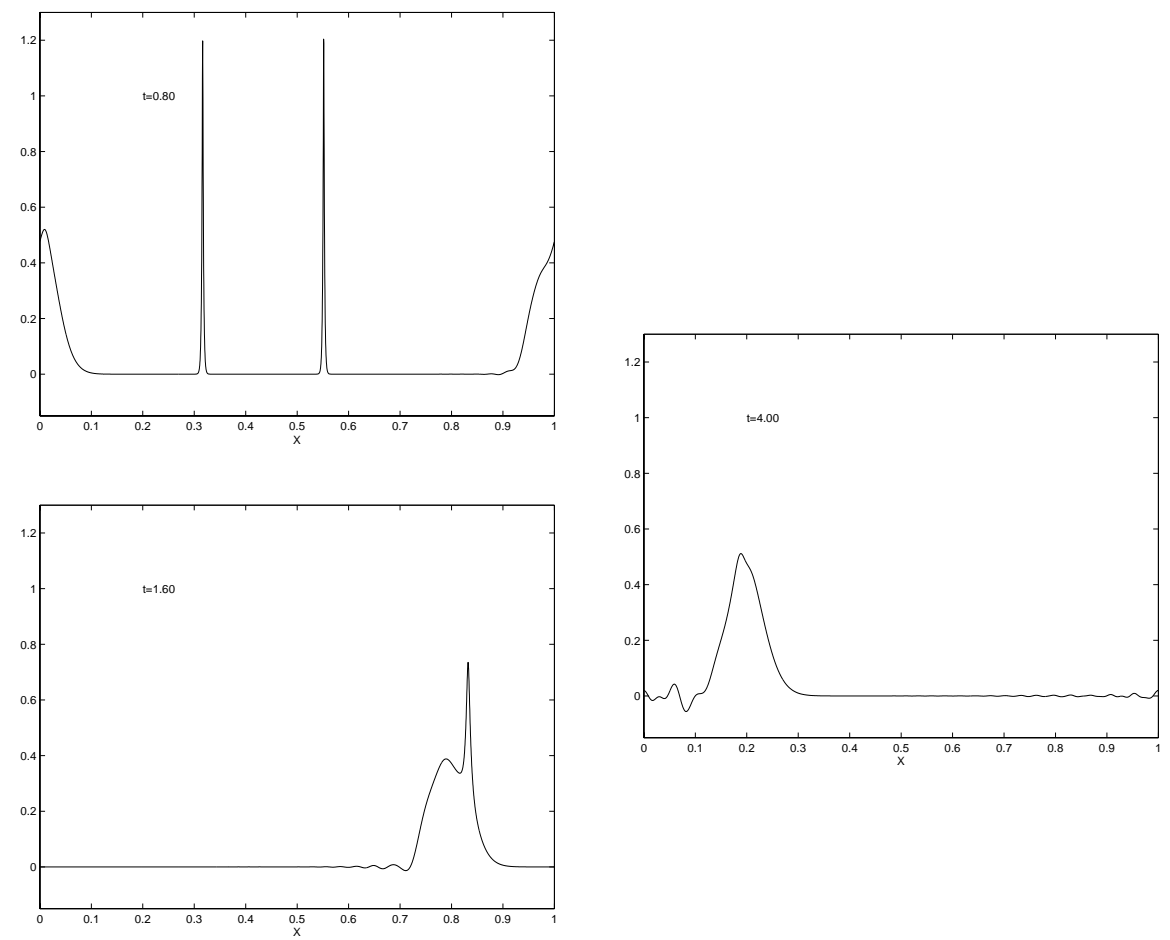

Fig. 20. Evolution of a Gaussian pulse, $p=8$. (a) At $t=0.8$. (b) After the solitary-wave structures appearing in (a) were removed. (c) After the solitary-wave structure in (b) was removed.
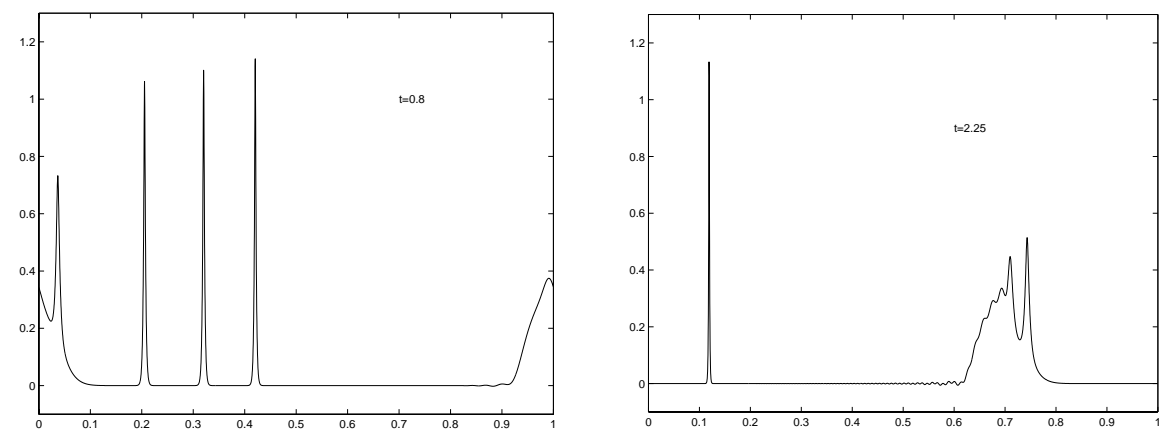

Fig. 21. Evolution of a Gaussian pulse, $p=6$. (a) At $t=0.80$. (b) At $t=2.25$ after the three isolated solitary-waves shown in (a) have been subtracted.

\section{References}

[1] D. H. Peregrine, "Calculations of the development of an undular bore," J. Fluid Mechanics 25 (1996), 321-330.

[2] D. H. Peregrine, "Long waves on a beach," J. Fluid Mechanics 27 (1967), 815-827.

[3] T. B. Benjamin, J. L. Bona, \& J. J. Mahony, "Model equations for long waves in nonlinear dispersive systems," Phil. Trans. Royal Soc. London, A 227 (1972), 47-78. 
[4] J. L. Bona, W. G. Pritchard, \& L. R. Scott, "A comparison of solutions of two model equations for long waves," in Fluid Dynamics in Astrophysics and Geophysics, Norman R. Lebovitz, ed., Lectures in Applied Mathematics \#20, 1983, 235-267.

[5] J. L. Bona, W. G. Pritchard, \& L. R. Scott, "An evaluation of a model equation for water waves," Phil. Trans. Royal Soc. London, A 302 (1981), 457-510.

[6] J. C. Eilbeck \& G. R. McGuire, "Numerical study of the regularized long wave equation I: Numerical methods," J. Computational Physics 19 (1975), 63-73.

[7] B.-Y. Guo \& V. S. Manoranjan, "Spectral method for solving the RLW equation," J. Computational Math. 3 (1985), 228-237.

[8] J. L. Bona, W. G. Pritchard, \& L. R. Scott, "Numerical schemes for a model for nonlinear dispersive waves," J. Computational Physics 60 (1985), 167-186.

[9] J. B. McLeod \& P. J. Olver, "The connection between completely integrable partial differential equations and ordinary differential equations of Painlevé type," SIAM J. Math Analysis 14 (1983), 56-75.

[10] J. Tasi, "Evolution of shockwaves in a one-dimensional lattice," J. Applied Physics 51 (1980), $5804-5815$.

[11] J. Tasi, "A second-order Korteweg-de Vries equation for a lattice," J. Applied Physics 51 (1980), 5816-5827.

[12] T. B. Benjamin, "Lectures on Nonlinear Wave Motion," in Nonlinear Wave Motion, Alan Newell, ed., Lectures in Applied Mathematics \#15, American Math. Soc., Providence, 1974, 3-47.

[13] T. B. Benjamin, “A new kind of solitary wave," J. Fluid Mechanics 245 (1992), 401-411.

[14] T. B. Benjamin, "Solitary and periodic waves of a new kind," Phil. Trans. Royal Soc. London, A 354 (1996), 1775-1806.

[15] J.-C. Saut, "Sur quelques généralisations de l'équation de Korteweg-de Vries," J. Math. Pures Appl. 58 (1979), 21-61.

[16] J.-C. Saut, "Quelques Généralisations de l'Équation de Korteweg-de Vries, II," J. IntegroDifferential Eq. 33 (1979), 320-335.

[17] J. P. Albert, J. L. Bona, \& J. M. Restrepo, "Solitary-wave solutions of the Benjamin equation," SIAM J. Applied Math. 59 (1999), 2139-2161.

[18] J. L. Bona, "Solitary waves and other phenomena associated with model equations for long waves," Fluid Dynamics Trans. 10 (1980), 77-111.

[19] J. L. Bona, "On solitary waves and their role in the evolution of long waves," in Applications of Nonlinear Analysis in the Physical Sciences, H. Amann, N. Bazley, K. Kirchgässner, ed., Pitman Press, London, 1981, 183-205.

[20] L. Abdelouhab, J. L. Bona, M. Felland, \& J.-C. Saut, "Nonlocal models for nonlinear dispersive waves," Physica D 40 (1989), 360-392.

[21] M. J. Ablowitz \& H. Segur, Solitons and the Inverse Scattering Transform, Society for Industrial and Applied Mathematics, Philadelphia, PA, 1981.

[22] J. L. Bona, W. G. Pritchard, \& L. R. Scott, "Solitary-wave interaction," Physics of Fluids 23 (1980), 438-441.

[23] J. L. Bona \& A. Soyeur, "On the stability of solitary-wave solutions for model equations for long waves," J. Nonlinear Sci. 4 (1994), 449-470.

[24] M. I. Weinstein, "Existence and dynamic stability of solitary-wave solutions of equations arising in long-wave propagation," Comm. Partial Differential Eq. 12 (1987), 11331173.

[25] T. B. Benjamin, J. L. Bona, \& D. K. Bose, "Solitary-wave solutions of nonliner problems," Phil. Trans. Royal Soc. London, A 340 (1990), 195-244.

[26] J. P. Albert, J. L. Bona, \& J.-C. Saut, "Model equations for stratified fluids," Proc. Royal Soc. London, A 453 (1997), 1233-1260.

[27] J. P. Albert, "Concentration compactness and the stability of solitary-wave solutions to nonlocal equations," in Contemporary Mathematics \#221, J. R. Dorroh, G. R. Goldstein, J. A. Goldstein \& M. M. Tom, ed., American Math. Soc., Providence, 1999, 1-29.

[28] J. Angulo, "Existence and stability of solitary wave solutions for the Benjamin equation," $J$. Differential Eq. 152 (1999), 136-159. 
[29] H. Chen \& J. L. Bona, "Existence and asymptotic properties of solitary-wave solutions of Benjamin-type equations," Adv. Differential Eq. 3 (1998), 51-84.

[30] P. D. Lax, "Integrals of nonlinear equations of evolution and solitary waves," Comm. Pure Appl. Math. 21 (1968), 467-490.

[31] T. B. Benjamin, "The stability of solitary waves," Proc. Royal Soc. London, A 328 (1972), 153-183.

[32] J. L. Bona, "On the stability theory of solitary waves," Proc. Royal Soc. London, A 344 (1975), 363-374.

[33] J. L. Bona, P. E. Souganidis, \& W. A. Strauss, "Stability and instability of solitary waves of Korteweg-de Vries type," Proc. Royal Soc. London, A 411 (1987), 395-412.

[34] J. L. Bona, V. A. Dougalis, O. A. Karakashian, \& W. R. McKinney, "Numerical simulation of singular solutions of the generalized Korteweg-de Vries equation," in Contemporary Mathematics \#200, F. Dias, J.-M. Ghidaglia, J.-C. Saut, ed., American Math. Soc., Providence, 1996, 17-29.

[35] B. Fornberg \& G. B. Whitham, "A numerical and theoretical study of certain nonlinear wave phenomena," Phil. Trans. Royal Soc. London, A 289 (1978), 373-404.

[36] R. L. Pego \& M. I. Weinstein, "Asymptotic stability of solitary waves," Comm. Math. Phys. 164 (1994), 305-349.

[37] J. L. Bona, V. A. Dougalis, O. A. Karakashian, \& W. R. McKinney, "Conservative, high-order numerical schemes for the generalized Korteweg-de Vries equation," Phil. Trans. Royal Soc. London, A 351 (1995), 107-164.

[38] Y. Liu, "Instability and blow-up of solutions to generalized Boussinesq equations," SIAM J. Math. Anal. 26 (1995), 1527-1546.

[39] Y. Liu, "Strong instability of solitary-wave solutions of a generalized Boussinesq equation," J. Differential Eq. 164 (2000), 223-239.

[40] J. P. Albert, J. L. Bona, \& D. Henry, "Sufficient conditions for stability of solitary-wave solutions of model equations for long waves," Physica D 24 (1987), 343-366.

[41] J. R. Miller \& M. I. Weinstein, "Asymptotic stability of solitary waves for the regularized long-wave equation," Comm. Pure Appl. Math. 49 (1996), 299-441.

[42] P. E. Souganidis \& W. A. Strauss, "Instability of a class of dispersive solitary waves," Proc. Royal Soc. Edinburgh 114A (1990), 195-212.

[43] D. M. Sloan, "Fourier pseudospectral solution of the regularised long wave equation," $J$. Computational and Applied Math. 36 (1991), 159-179.

[44] J. Pasciak, "Spectral methods for a nonlinear initial value problems involving pseudodifferential operators," SIAM J. Numer. Anal. 19 (1982), 142-154.

[45] J. L. Bona, "Convergence of periodic wavetrains in the limit of large wavelength," Applied Science Research 37 (1981), 21-30.

[46] C. Canuto, M. Y. Hussaini, A. Quarteroni, \& T. A. Zang, Spectral Methods in Fluid Dynamics, Springer-Verlag, New York-Heidelberg-Berlin, 1988.

[47] P. N. Swartztrauber, NCAR Software, available at www . net lib.org, 1989.

[48] L. F. Shampine \& M. K. Gordon, Computer Solution of Ordinary Differential Equations: The Initial Value Problem, W. H. Freeman, San Francisco, 1975.

[49] I. S. Gradshteyn \& I. M. Rysik, Table of Integrals, Series, and Products, Academic Press, New York, 1980.

[50] J. P. Albert, "Dispersion of low-energy waves for the generalized Benjamin-Bona-Mahony equation," J. Differential Eq. 63 (1986), 117-134.

[51] J.P. Albert, "On the decay of solutions of the generalized Benjamin-Bona-Mahony equation," J. Math. Anal. Appl. 141 (1989), 527-537. 